\title{
EXISTENCE RESULTS FOR SUPERLINEAR ELLIPTIC EQUATIONS WITH NONLINEAR BOUNDARY VALUE CONDITIONS
}

\author{
XIAOHUI YU*
}

\begin{abstract}
In this paper, we study the existence of solutions for the following superlinear elliptic equation with nonlinear boundary value condition

$$
\begin{cases}-\Delta u+u=|u|^{r-2} u & \text { in } \Omega, \\ \frac{\partial u}{\partial \nu}=|u|^{q-2} u & \text { on } \partial \Omega,\end{cases}
$$
\end{abstract}

where $\Omega \subset \mathbb{R}^{N}, N \geq 3$ is a bounded domain with smooth boundary. We will prove the existence results for the above equation under four different cases: (i) Both $q$ and $r$ are subcritical; (ii) $r$ is critical and $q$ is subcritical; (iii) $r$ is subcritical and $q$ is critical; (iv) Both $q$ and $r$ are critical.

keywords: existence result, critical exponent, trace inequality, nonlinear boundary value, (PS) condition.

Mathematics Subject Classification (2010): 35J60, 35J57, 35J15.

\section{INTRODUCTION}

In this paper, we study the existence of solutions for the following superlinear elliptic equation with nonlinear boundary value condition

$$
\begin{cases}-\Delta u+u=|u|^{r-2} u & \text { in } \Omega, \\ \frac{\partial u}{\partial \nu}=|u|^{q-2} u & \text { on } \partial \Omega,\end{cases}
$$

where $\Omega \subset \mathbb{R}^{N}, N \geq 3$ is a bounded domain with smooth boundary. We also assume that $r, q>2$ so that this problem is a superlinear one.

Nonlinear boundary value problems were widely studied in the past few decades and there are many results on this aspect. For example, Y.Li and M.Zhu [19] classified all the positive solutions for equation

$$
\begin{cases}-\Delta u=|u|^{2^{*}-2} u & \text { in } \mathbb{R}_{+}^{N}, \\ \frac{\partial u}{\partial \nu}=|u|^{2_{*}-2} u & \text { on } \partial \mathbb{R}_{+}^{N},\end{cases}
$$

where $2^{*}=\frac{2 N}{N-2}$ is the usual critical Sobolev exponent and $2_{*}=\frac{2(N-1)}{N-2}$ is the critical exponent for Sobolev trace inequality. They proved the positive solutions of problem (1.2) must have the following form

$$
u_{\varepsilon}(x)=\frac{[N(N-2)]^{\frac{N-2}{4}} \varepsilon^{\frac{N-2}{2}}}{\left[\varepsilon^{2}+\left|x^{\prime}\right|^{2}+\left(x_{N}+\varepsilon x_{N}^{0}\right)^{2}\right]^{\frac{N-2}{2}}},
$$

*The Center for China's Overseas Interests, Shenzhen University, Shenzhen Guangdong, 518060, People's Republic of China(yuxiao_211@163.com). 
where $x^{\prime}=\left(x_{1}, x_{2}, \cdots, x_{N-1}\right), x_{N}^{0}=\left(\frac{N}{N-2}\right)^{\frac{1}{2}}$ is a positive constant depending only on $N$. The main method in this paper is the moving sphere method. Similar results can be found in [10]. Later, by using the moving sphere method and Harnack inequality, Y.Li and L.Zhang [18] studied the Liouville type theorems for elliptic equations with nonlinear boundary value conditions. X.Yu [24] studied the nonexistence results for nonlinear boundary value problems with general nonlinearities. B.Hu [12] studied the nonexistence results of harmonic function with nonlinear boundary condition. Other results can be found in [13] 15] 16 .

On the other hand, existence results for nonlinear boundary value problems were also widely studied. For example, M.Chipota, M.Chlebik, M.Fila and I.Shafrir [9] studied the existence results for nonlinear boundary value problem in $\mathbb{R}_{+}^{N}$. J.Bonder and J.Rossi [2] studied the existence results for nonlinear boundary value problems involving $p$-Laplacian operator. In order to overcome the difficulty of nonlocal property of the fractional Laplacian operators, X.Cabre and J.Tan [6] transformed the fractional Laplacian equations into nonlinear boundary value problems by the extension theorem in [7]. Then they studied the existence results, regularity results, nonexistence results for fractional Laplacian equations. Later, J.Tan [21] studied the existence result for critical fractional Laplacian equations. He still turned the problem into a nonlinear boundary value problem. Other results on fractional Laplacian equations can be found in [1] [3] [17] and etc.

In this paper, we study the existence results for nonlinear boundary value problem (1.1). This equation involves two nonlinear terms. We first consider the case where both $r$ and $q$ are subcritical. We have the following multiple solutions result.

Theorem 1.1. Suppose that the nonlinear terms are superlinear and subcritical, i.e., $2<$ $r<\frac{2 N}{N-2}$ and $2<q<\frac{2(N-1)}{N-2}$, then problem (1.1) has infinitely many solutions.

However, if $r$ or(and) $q$ is critical, things become more difficult. The main difficulty of solving the problem by critical point theory lies in that the Sobolev embedding or(and) the Sobolev trace embedding is not compact, then the so called Palais-Smale condition is generally not satisfied by the related functional $I$. The most significant achievement in this aspect is the work [5] in which the authors studied the existence result of the following problem

$$
\begin{cases}-\Delta u=|u|^{2^{*}-2} u+\lambda u & \text { in } \Omega \\ u=0 & \text { on } \partial \Omega .\end{cases}
$$

They first proved the corresponding functional satisfies the $(P S)_{c}$ condition for $c \in\left(0, \frac{1}{N} S^{\frac{N}{2}}\right)$, where $S$ is the best constant of $D^{1,2}\left(\mathbb{R}^{N}\right) \hookrightarrow L^{2^{*}}\left(\mathbb{R}^{N}\right)$. Then they proved the Mountain Pass level of the corresponding functional indeed belongs to this interval under some assumptions on $\lambda$. Then the existence result was obtained. After the work [5], there were plenty of works on critical Laplacian equations and we can't list all of them. For example, X.Wang [22] studied the existence results for critical Neumann boundary value problem. J.Tan [21] studied the existence result for critical fractional Laplacian equations in bounded domain. B.Barrios, E.Colorado, A.de Pablo and U.Sánchez [1] studied the existence result 
for a more general critical fractional Laplacian equation. Hua and $\mathrm{Yu}$ [17] studied the existence result for critical fractional Laplacian equations under other circumstance. Ye and $\mathrm{Yu}$ [23] studied the global compactness results for critical Laplacian equations in the whole spaces.

Inspired by the above works, we next consider the case where $q$ is subcritical and $r$ is the critical exponent. We have the following existence result.

Theorem 1.2. Suppose that $r=\frac{2 N}{N-2}$ and $2<q<\frac{2(N-1)}{N-2}$, then problem (1.1) has at least one nontrivial solution.

Similarly, we can study the case where $r$ is subcritical and $q$ is critical. For this case, we have the following existence result.

Theorem 1.3. Suppose that $2<r<\frac{2 N}{N-2}$ and $q=\frac{2(N-1)}{N-2}$, then problem (1.1) has at least one nontrivial solution.

Finally, we study the most difficult case in which both $q$ and $r$ are critical. We have the following existence result.

Theorem 1.4. Suppose that $r=\frac{2 N}{N-2}$ and $q=\frac{2(N-1)}{N-2}$, then problem (1.1) possesses at least one nontrivial solution.

The proof of the above theorems is based on the variational methods. Obiviously, the solutions of problem (1.1) correspond to the critical points of the functional

$$
I(u)=\frac{1}{2} \int_{\Omega}|\nabla u|^{2}+u^{2} d x-\frac{1}{r} \int_{\Omega}|u|^{r} d x-\frac{1}{q} \int_{\partial \Omega}|u|^{q} d S
$$

defined on $H^{1}(\Omega)$. According to the well-known Sobolev inequality and Sobolev trace inequality, we know that the functional $I$ is well-defined and is of $C^{2}$ class. If both $q$ and $r$ are subcritical, then the imbeddings $H^{1}(\Omega) \hookrightarrow L^{r}(\Omega)$ and $H^{1}(\Omega) \hookrightarrow L^{q}(\partial \Omega)$ are compact. So it is easy to verify that $I$ satisfies the usual $(P S)$ condition. That is, if $I\left(u_{n}\right)$ is bounded and $I^{\prime}\left(u_{n}\right) \rightarrow 0$, then $u_{n}$ must have a convergent subsequence. However, if $r$ or $($ and $) q$ is critical, then the imbedding $H^{1}(\Omega) \hookrightarrow L^{r}(\Omega)$ or (and) $H^{1}(\Omega) \hookrightarrow L^{q}(\partial \Omega)$ is not compact. As a result, the functional $I$ does not satisfies the $(P S)$ condition. To overcome this difficulty, one usually uses the $(P S)_{c}$ condition to substitute the $(P S)$ condition. This method has been widely used in dealing with elliptic equation involving critical exponent, see [5] [17] [21] and etc. The spirit of this paper is the same as the above works. We use $(P S)_{c}$ condition to substitute the usual $(P S)$ condition. However, due to the different cases of $q$, $r$, we need to find different intervals so that $I$ satisfies the $(P S)_{c}$ condition for $c$ in those intervals. More precisely, if $r=\frac{2 N}{N-2}$ and $q<\frac{2(N-1)}{N-2}$, we will show that $I$ satisfies the $(P S)_{c}$ condition for $c \in\left(0, \frac{1}{2 N} S^{\frac{N}{2}}\right)$, where $S$ is the usual Sobolev constant defined by

$$
S=\inf _{u \in D^{1,2}\left(\mathbb{R}^{N}\right), u \neq 0} \frac{\|\nabla u\|_{L^{2}\left(\mathbb{R}^{N}\right)}^{2}}{\|u\|_{L^{2^{*}}\left(\mathbb{R}^{N}\right)}^{2}}
$$


However, for the case $r<\frac{2 N}{N-2}$ and $q=\frac{2(N-1)}{N-2}$, we will show that $I$ satisfies the $(P S)_{c}$ condition for $c \in\left(0, \frac{1}{2(N-1)} S_{T}^{N-1}\right)$, where $S_{T}$ is the best constant of Sobolev trace inequality which is defined by

$$
S_{T}=\inf _{u \in D^{1,2}\left(\mathbb{R}_{+}^{N}\right), u \neq 0} \frac{\|\nabla u\|_{L^{2}\left(\mathbb{R}_{+}^{N}\right)}^{2}}{\left\|u\left(x^{\prime}, 0\right)\right\|_{L^{2 *}\left(\partial \mathbb{R}_{+}^{N}\right)}^{2}} .
$$

The most difficult problem is the case in which both $r$ and $q$ are critical, we will show that the functional $I$ satisfies the $(P S)_{c}$ condition for $c \in\left(0, c_{\infty}\right)$, where $c_{\infty}$ is the ground state level of equation (1.2). In [19], the authors proved that $c_{\infty}$ can be attained by some functions and they gave all the expressions of these functions.

After the $(P S)_{c}$ condition is proved, then we need to show that the Mountain Pass level of the functional $I$ indeed belongs to these intervals. Then the standard Mountain Pass Theorem implies the above theorems.

The rest of this paper is devoted to the proof of the above theorems. In Section 2, we establish the multiple solutions result for the subcritical problem, i.e., we prove Theorem 1.1. We guess this result is well-known but we can't find the proper reference so we give its proof to keep this paper self-contained. In Section 3,4,5, we prove Theorem 1.2, Theorem 1.3 and Theorem 1.4 respectively. In the following, we denote $C$ by a positive constant, which may vary from line to line.

\section{Proof of Theorem 1.1}

In this section, we study the multiple solutions of problem (1.1) under the subcritical assumptions, i.e., $2<r<\frac{2 N}{N-2}$ and $2<q<\frac{2(N-1)}{N-2}$. We need the following technical lemma, see Theorem 9.12 in [20].

Lemma 2.1. Let $E$ be an infinite dimensional Banach space and let $I \in C^{2}(E, \mathbb{R})$ be even, satisfying $(P S)$, and $I(0)=0$. If $E=V \oplus X$, where $V$ is finite dimensional, and $I$ satisfies (i) there exist constants $\rho, \alpha>0$, such that $I_{\partial B_{\rho} \cap X} \geq \alpha$, and

(ii) for each finite dimensional subspace $\tilde{E} \subset E$, there is an $R=R(\tilde{E})$ such that $I \leq 0$ on $\tilde{E} \backslash B_{R(\tilde{E})}$,

then I possesses an unbounded sequence of critical values.

To prove Theorem 1.1, we only need to verify that $I$ satisfies the conditions in Lemma 2.1 under the assumptions of Theorem 1.1. This is composed of the following lemmas. We first show that $I$ satisfies the $(P S)$ condition.

Lemma 2.2. Suppose that $2<r<\frac{2 N}{N-2}$ and $2<q<\frac{2(N-1)}{N-2}$, then I satisfies the (PS) condition.

Proof. Let $\left\{u_{n}\right\}$ be a (PS) sequence, that is $\left|I\left(u_{n}\right)\right|$ is bounded and $I^{\prime}\left(u_{n}\right) \rightarrow 0$, we need to show that $\left\{u_{n}\right\}$ has a convergent subsequence.

We first show that $\left\{u_{n}\right\}$ is bounded. By means of $(P S)$ sequence, we have

$$
\frac{1}{2} \int_{\Omega}\left|\nabla u_{n}\right|^{2}+u_{n}^{2} d x-\frac{1}{r} \int_{\Omega}\left|u_{n}\right|^{r} d x-\frac{1}{q} \int_{\partial \Omega}\left|u_{n}\right|^{q} d S \leq C
$$


and

$$
\int_{\Omega}\left|\nabla u_{n}\right|^{2}+u_{n}^{2} d x-\int_{\Omega}\left|u_{n}\right|^{r} d x-\int_{\partial \Omega}\left|u_{n}\right|^{q} d S=o(1)\left\|u_{n}\right\|
$$

If $r \geq q$, then we infer from the above two equations that

$$
\left(\frac{1}{2}-\frac{1}{q}\right)\left\|u_{n}\right\|^{2}+\left(\frac{1}{q}-\frac{1}{r}\right) \int_{\Omega}\left|u_{n}\right|^{r} d x \leq C+o(1)\left\|u_{n}\right\|
$$

which implies that $\left\|u_{n}\right\|$ is bounded. On the other hand, if $r<q$, then we can still deduce from equation (2.1) and equation (2.2) that

$$
\left(\frac{1}{2}-\frac{1}{r}\right)\left\|u_{n}\right\|^{2}+\left(\frac{1}{r}-\frac{1}{q}\right) \int_{\partial \Omega}\left|u_{n}\right|^{q} d S \leq C+o(1)\left\|u_{n}\right\|,
$$

which also implies that $\left\|u_{n}\right\|$ is bounded. So we proved that $\left\{u_{n}\right\}$ is bounded.

Next, we show that $\left\{u_{n}\right\}$ has a convergent subsequence. Since $\left\{u_{n}\right\}$ is bounded, we can suppose that, up to a subsequence, $u_{n} \rightarrow u$. Then the Sobolev compact imbedding theorem implies that

$$
\int_{\Omega}\left|u_{n}\right|^{r} d x \rightarrow \int_{\Omega}|u|^{r} d x
$$

and

$$
\int_{\partial \Omega}\left|u_{n}\right|^{q} d S \rightarrow \int_{\partial \Omega}|u|^{q} d S
$$

Hence, we deduce from equation (2.2) that

$$
\left\|u_{n}\right\|^{2} \rightarrow \int_{\Omega}|u|^{r} d x+\int_{\partial \Omega}|u|^{q} d S=\|u\|^{2} .
$$

Since $u_{n} \rightarrow u$ and $\left\|u_{n}\right\| \rightarrow\|u\|$, we get that $u_{n} \rightarrow u$ strongly in $H^{1}(\Omega)$. This proves this lemma.

Let $V=\emptyset$ and $E=X=H^{1}(\Omega)$ in Lemma 2.1, our next lemma shows that (i) of Lemma 2.1 holds.

Lemma 2.3. There exist constants $\rho, \alpha>0$, such that $I_{\partial B_{\rho} \cap X} \geq \alpha$.

Proof. By the Sobolev embedding theorem and Sobolev trace inequality, we deduce that

$$
I(u) \geq \frac{1}{2}\|u\|^{2}-C\|u\|^{r}-C\|u\|^{q} .
$$

Hence, we can choose $\alpha, \rho>0$ small enough, such that

$$
I_{\partial B_{\rho} \cap X} \geq \alpha>0 \text {. }
$$

Finally, we verify condition (ii) in Lemma 2.1, we have the following result.

Lemma 2.4. Let $\tilde{E} \subset H^{1}(\Omega)$ be a finite dimensional subspace, then there exists $R=R(\tilde{E})$ such that $I \leq 0$ on $\tilde{E} \backslash B_{R(\tilde{E})}$. 
Proof. Since $\tilde{E}$ is finite dimensional, then any norms on $\tilde{E}$ are equivalent. So we have

$$
I(u) \leq C_{1}\|u\|^{2}-C_{2}\|u\|^{r}-C_{3}\|u\|^{q}
$$

for any $u \in \tilde{E}$. Moreover, we deduce from $2<r<\frac{2 N}{N-2}$ and $2<q<\frac{2(N-1)}{N-2}$ that there exists $R=R(\tilde{E})>0$ such that

$$
I(u)<0
$$

on $\tilde{E} \backslash B_{R(\tilde{E})}$.

Proof of Theorem 1.1: It is easy to see that $I \in C^{2}\left(H^{1}(\Omega), \mathbb{R}\right)$ is even. Moreover, we infer from Lemma 2.2. Lemma 2.3 and Lemma 2.4 that $I$ satisfies all the conditions in Lemma 2.1. So we conclude that problem (1.1) possesses infinitely many solutions under the assumptions of Theorem 1.1 .

\section{Proof of Theorem 1.2}

In this section, we study the existence result for problem (1.1) under the assumption that $r$ is the critical exponent and $q$ is subcritical. We first have the following local compactness result.

Lemma 3.1. Suppose that $r=\frac{2 N}{N-2}$ and $2<q<\frac{2(N-1)}{N-2}$, then I satisfies the $(P S)_{c}$ condition for $c \in\left(0, \frac{1}{2 N} S^{\frac{N}{2}}\right)$, where $S$ is the best constant of Sobolev inequality defined by

$$
S=\inf _{u \in D^{1,2}\left(\mathbb{R}^{N}\right), u \neq 0} \frac{\|\nabla u\|_{L^{2}\left(\mathbb{R}^{N}\right)}^{2}}{\|u\|_{L^{2^{*}}\left(\mathbb{R}^{N}\right)}^{2}} .
$$

Proof. Let $\left\{u_{n}\right\} \subset H^{1}(\Omega)$ be a $(P S)_{c}$ sequence for $I$ with $c \in\left(0, \frac{1}{2 N} S^{\frac{N}{2}}\right)$, that is,

$$
I\left(u_{n}\right) \rightarrow c
$$

and

$$
I^{\prime}\left(u_{n}\right) \rightarrow 0
$$

we need to show that $\left\{u_{n}\right\}$ has a convergent subsequence.

We first show that $\left\{u_{n}\right\}$ is bounded. In fact, we get from the above two equations that

$$
\frac{1}{2} \int_{\Omega}\left|\nabla u_{n}\right|^{2}+u_{n}^{2} d x-\frac{1}{2^{*}} \int_{\Omega}\left|u_{n}\right|^{2^{*}} d x-\frac{1}{q} \int_{\partial \Omega}\left|u_{n}\right|^{q} d S \rightarrow c
$$

and

$$
\int_{\Omega}\left|\nabla u_{n}\right|^{2}+u_{n}^{2} d x-\int_{\Omega}\left|u_{n}\right|^{2^{*}} d x-\int_{\partial \Omega}\left|u_{n}\right|^{q} d S=o(1)\left\|u_{n}\right\| .
$$

Moreover, the above two equations imply that

$$
\left(\frac{1}{2}-\frac{1}{q}\right)\left\|u_{n}\right\|^{2}+\left(\frac{1}{q}-\frac{1}{2^{*}}\right) \int_{\Omega}\left|u_{n}\right|^{2^{*}} d x=c+o(1)+o(1)\left\|u_{n}\right\|,
$$

which implies that $\left\|u_{n}\right\|$ is bounded. 
Next, we show that $\left\{u_{n}\right\}$ has a convergent subsequence. Since $\left\{u_{n}\right\}$ is bounded, then we can suppose that, up to a subsequence, $u_{n} \rightarrow u$ in $H^{1}(\Omega), u_{n} \rightarrow u$ in $L^{2^{*}}(\Omega), u_{n} \rightarrow u$ in $L^{2}(\Omega)$ and $u_{n} \rightarrow u$ in $L^{q}(\partial \Omega)$. Now let $v_{n}=u_{n}-u$, then we deduce from Brezis-Lieb theorem [4] that

$$
\frac{1}{2} \int_{\Omega}\left|\nabla v_{n}\right|^{2} d x-\frac{1}{2^{*}} \int_{\Omega}\left|v_{n}\right|^{2^{*}} d x=c-I(u)+o(1)
$$

and

$$
\int_{\Omega}\left|\nabla v_{n}\right|^{2} d x-\int_{\Omega}\left|v_{n}\right|^{2^{*}} d x=o(1) .
$$

Hence, we get

$$
\int_{\Omega}\left|\nabla v_{n}\right|^{2} d x=\int_{\Omega}\left|v_{n}\right|^{2^{*}} d x=N(c-I(u))+o(1)
$$

Obviously, to prove that $u_{n} \rightarrow u$, it is equivalent to prove that $v_{n} \rightarrow 0$ in $H^{1}(\Omega)$. We prove this conclusion by contradiction. Suppose on the contrary, that is, $v_{n} \not \rightarrow 0$ in $H^{1}(\Omega)$, then we will show that this will lead to a contradiction.

Let $\varepsilon$ be any fixed positive constant, then by Lemma 2.1 in [22], there exists $\delta=\delta(\varepsilon)>0$, such that if $\operatorname{diam}(\operatorname{supp}\{\varphi\}) \leq \delta$, then

$$
\int_{\Omega}|\nabla \varphi|^{2} d x \geq\left(2^{-\frac{2}{N}} S-\varepsilon\right)\left[\int_{\Omega}|\varphi|^{2^{*}} d x\right]^{\frac{2}{2^{*}}}
$$

where $\operatorname{diam}(D)$ is the diameter of the set $D$. Now let $\left\{\varphi_{\alpha}\right\}_{\alpha=1}^{n_{0}}$ be a unit partition of $\bar{\Omega}$ with $\operatorname{diam}\left(\operatorname{supp}\left\{\varphi_{\alpha}\right\}\right) \leq \delta$ for each $\alpha$. Since $\partial \Omega \in C^{1}$, then we deduce from Lemma 2.1 in [22] that

$$
\int_{\Omega}\left|\nabla\left(u \varphi_{\alpha}\right)\right|^{2} d x \geq\left(2^{-\frac{2}{N}} S-\varepsilon\right)\left[\int_{\Omega}\left|u \varphi_{\alpha}\right|^{2^{*}} d x\right]^{\frac{2}{2^{*}}}
$$


Hence, we have

$$
\begin{aligned}
{[N(c-I(u)+o(1))]^{\frac{2}{2^{*}}} } & =\left(\int_{\Omega}\left(v_{n}\right)^{2^{*}} d x\right)^{\frac{2}{2^{*}}} \\
& =\left\|v_{n}^{2}\right\|_{L^{\frac{2^{*}}{2}}(\Omega)} \\
& =\left\|\sum_{\alpha=1}^{n_{0}} \varphi_{\alpha} v_{n}^{2}\right\|_{L^{\frac{2^{*}}{2}}(\Omega)} \\
& \leq \sum_{\alpha=1}^{n_{0}}\left\|\varphi_{\alpha} v_{n}^{2}\right\|_{L^{\frac{2^{*}}{2}}(\Omega)} \\
& \leq\left(2^{-\frac{2}{N}} S-\varepsilon\right)^{-1} \sum_{\alpha=1}^{n_{0}} \int_{\Omega}\left|\nabla\left(v_{n} \varphi_{\alpha}^{\frac{1}{2}}\right)\right|^{2} d x \\
& \leq\left(2^{-\frac{2}{N}} S-\varepsilon\right)^{-1}\left[(1+\varepsilon) \int_{\Omega}\left|\nabla v_{n}\right|^{2} d x+C \int_{\Omega} v_{n}^{2} d x\right] \\
& =\left(2^{-\frac{2}{N}} S-\varepsilon\right)^{-1}(1+\varepsilon) \int_{\Omega}\left|\nabla v_{n}\right|^{2} d x+o(1) \\
& =\left(2^{-\frac{2}{N}} S-\varepsilon\right)^{-1}(1+\varepsilon)[N(c-I(u)+o(1))] .
\end{aligned}
$$

Moreover, we infer from the above inequality that

$$
[c-I(u)+o(1)] \geq \frac{1}{N}\left[\frac{2^{-\frac{2}{N}} S-\varepsilon}{1+\varepsilon}\right]^{\frac{N}{2}} .
$$

We note that $I(u) \geq 0$ since $u$ is a solution of problem (1.1). So if we let $\varepsilon \rightarrow 0$ and $n \rightarrow \infty$ in equation (3.6), then we get

$$
[c-I(u)] \geq \frac{1}{2 N} S^{\frac{N}{2}}
$$

which contradicts that $c \in\left(0, \frac{1}{2 N} S^{\frac{N}{2}}\right)$. This finishes the proof of this lemma.

We want to use the Mountain Pass theorem in [20] to prove the existence result. By the above local $(P S)$ condition, we need to prove that the Mountain Pass level of $I$ is indeed below $\frac{1}{2 N} S^{\frac{N}{2}}$. Since $\Omega$ is bounded, then there exists a ball $B_{R}(\bar{x})$ containing $\Omega$ and $\partial B_{R}(\bar{x}) \cap \bar{\Omega} \neq \emptyset$. Suppose that $x_{0} \in \partial B_{R}(\bar{x}) \cap \bar{\Omega}$, then we have $2 \alpha_{i} \geq \frac{1}{R}$ for each $1 \leq i \leq N-1$, where $2 \alpha_{i}(i=1, \cdots, N-1)$ are the principal curvatures of $\partial \Omega$ at $x_{0}$. We can suppose $x_{0}=0$ and $\Omega \subset\left\{x: x_{N}>0\right\}$ without loss of generality, then the boundary of $\Omega$ can be represented by

$$
x_{N}=h\left(x^{\prime}\right)=\sum_{i=1}^{N-1} \alpha_{i} x_{i}^{2}+o\left(\left|x^{\prime}\right|^{2}\right), \quad \forall x^{\prime}=\left(x_{1}, x_{2}, \cdots, x_{N-1}\right) \in D(0, \delta)
$$


EXISTENCE RESULTS FOR SUPERLINEAR ELLIPTIC EQUATIONS WITH NONLINEAR BOUNDARY VALUE CONDITIO for some $\delta>0$, where $D(0, \delta)=B_{\delta}(0) \cap\left\{x: x_{N}=0\right\}$. Set

$$
u_{\varepsilon}(x)=\frac{\left[N(N-2) \varepsilon^{2}\right]^{\frac{N-2}{4}}}{\left[\varepsilon^{2}+|x|^{2}\right]^{\frac{N-2}{2}}} \text { in } \mathbb{R}_{+}^{N},
$$

then it is well-known that $u_{\varepsilon}$ solves equation

$$
\begin{cases}-\Delta u=|u|^{2^{*}-2} u & \text { in } \mathbb{R}_{+}^{N} \\ \frac{\partial u}{\partial \nu}=0 & \text { on } \partial \mathbb{R}_{+}^{N} .\end{cases}
$$

Moreover, we have $\int_{\mathbb{R}_{+}^{N}}\left|\nabla u_{\varepsilon}\right|^{2} d x=\int_{\mathbb{R}_{+}^{N}} u_{\varepsilon}^{\frac{2 N}{N-2}} d x=\frac{1}{2} S^{\frac{N}{2}}$, where $S$ is the best constant of the Sobolev inequality.

With the above notations, we have the following estimates.

Lemma 3.2. Let $\Omega \subset \mathbb{R}^{N}$ with $N \geq 4$ as above, $u_{\varepsilon}$ be defined by equation (3.8) and $g\left(x^{\prime}\right)=\sum_{i=1}^{N-1} \alpha_{i} x_{i}^{2}$, then we have

(i) $\int_{\Omega}\left|\nabla u_{\varepsilon}\right|^{2} d x=\int_{\mathbb{R}_{+}^{N}}\left|\nabla u_{\varepsilon}\right|^{2} d x-N^{\frac{N-2}{2}}(N-2)^{\frac{N+2}{2}} \varepsilon \int_{\mathbb{R}^{N-1}} \frac{\left|x^{\prime}\right|^{2} g\left(x^{\prime}\right)}{\left[1+\left|x^{\prime}\right|^{2}\right]^{N}} d x^{\prime}+o(\varepsilon)$;

(ii) $\int_{\Omega} u_{\varepsilon}^{\frac{2 N}{N-2}} d x=\int_{\mathbb{R}_{+}^{N}} u_{\varepsilon}^{\frac{2 N}{N-2}}-N^{\frac{N}{2}}(N-2)^{\frac{N}{2}} \varepsilon \int_{\mathbb{R}^{N-1}} \frac{g\left(x^{\prime}\right)}{\left[1+\left|x^{\prime}\right|^{2}\right]^{N}} d x^{\prime}+o(\varepsilon)$;

(iii) $\int_{\partial \Omega} u_{\varepsilon}^{q}=\int_{\mathbb{R}^{N-1}} u_{\varepsilon}\left(x^{\prime}, 0\right)^{q} d x^{\prime}+o(\varepsilon)=C \varepsilon^{(N-1)-\frac{N-2}{2} q}+o\left(\varepsilon^{(N-1)-\frac{N-2}{2} q}\right)$ for $q \in\left(2,2_{*}\right)$;

(iv) $\int_{\Omega} u_{\varepsilon}^{2} d x= \begin{cases}O\left(\varepsilon^{2}|\ln \varepsilon|\right) & \text { if } N=4, \\ O\left(\varepsilon^{2}\right) & \text { if } N \geq 5 .\end{cases}$

Proof. A direct calculation implies that

$$
\begin{aligned}
& \int_{\mathbb{R}_{+}^{N}}\left|\nabla u_{\varepsilon}\right|^{2} d x-\int_{\Omega}\left|\nabla u_{\varepsilon}\right|^{2} d x \\
& =\int_{\mathbb{R}^{N-1}} d x^{\prime} \int_{0}^{g\left(x^{\prime}\right)} N^{\frac{N-2}{2}}(N-2)^{\frac{N+2}{2}} \varepsilon^{N-2} \frac{|x|^{2}}{\left[\varepsilon^{2}+|x|^{2}\right]^{N}} d x_{N} \\
& +\int_{D(0, \delta)} d x^{\prime} \int_{g\left(x^{\prime}\right)}^{h\left(x^{\prime}\right)} N^{\frac{N-2}{2}}(N-2)^{\frac{N+2}{2}} \varepsilon^{N-2} \frac{|x|^{2}}{\left[\varepsilon^{2}+|x|^{2}\right]^{N}} d x_{N}+O\left(\varepsilon^{N-2}\right) \\
& =\int_{\mathbb{R}^{N-1}} d x^{\prime} \int_{0}^{\varepsilon g\left(x^{\prime}\right)} N^{\frac{N-2}{2}}(N-2)^{\frac{N+2}{2}} \frac{|x|^{2}}{\left[1+|x|^{2}\right]^{N}} d x_{N} \\
& +\int_{D(0, \delta)} d x^{\prime} \int_{g\left(x^{\prime}\right)}^{h\left(x^{\prime}\right)} N^{\frac{N-2}{2}}(N-2)^{\frac{N+2}{2}} \varepsilon^{N-2} \frac{|x|^{2}}{\left[\varepsilon^{2}+|x|^{2}\right]^{N}} d x_{N}+O\left(\varepsilon^{N-2}\right) \\
& =N^{\frac{N-2}{2}}(N-2)^{\frac{N+2}{2}} \varepsilon \int_{\mathbb{R}^{N-1}} \frac{\left|x^{\prime}\right|^{2} g\left(x^{\prime}\right)}{\left[1+\left|x^{\prime}\right|^{2}\right]^{N}} d x^{\prime}+o(\varepsilon) \\
& +\int_{D(0, \delta)} d x^{\prime} \int_{g\left(x^{\prime}\right)}^{h\left(x^{\prime}\right)} N^{\frac{N-2}{2}}(N-2)^{\frac{N+2}{2}} \varepsilon^{N-2} \frac{|x|^{2}}{\left[\varepsilon^{2}+|x|^{2}\right]^{N}} d x_{N} .
\end{aligned}
$$


On the other hand, since $h\left(x^{\prime}\right)=g\left(x^{\prime}\right)+o\left(\left|x^{\prime}\right|^{2}\right)$ in $D(0, \delta)$, then it follows that $\forall \sigma>0$, there exists $C(\sigma)>0$ such that $\left|h\left(x^{\prime}\right)-g\left(x^{\prime}\right)\right| \leq \sigma\left|x^{\prime}\right|^{2}+C(\sigma)\left|x^{\prime}\right|^{\frac{5}{2}}$ for $x^{\prime} \in D(0, \delta)$. Therefore, we have

$$
\begin{aligned}
& \int_{D(0, \delta)} d x^{\prime} \int_{g\left(x^{\prime}\right)}^{h\left(x^{\prime}\right)} N^{\frac{N-2}{2}}(N-2)^{\frac{N+2}{2}} \varepsilon^{N-2} \frac{|x|^{2}}{\left[\varepsilon^{2}+|x|^{2}\right]^{N}} d x_{N} \\
\leq & C \varepsilon^{N-2} \int_{D(0, \delta)} d x^{\prime} \int_{g\left(x^{\prime}\right)}^{h\left(x^{\prime}\right)} \frac{|x|^{2}}{\left[\varepsilon^{2}+|x|^{2}\right]^{N}} d x_{N} \\
\leq & C \varepsilon^{N-2} \int_{D(0, \delta)} \frac{\left|h\left(x^{\prime}\right)-g\left(x^{\prime}\right)\right|}{\left[\varepsilon^{2}+\left|x^{\prime}\right|^{2}\right]^{N-1}} d x^{\prime} \\
\leq & C \varepsilon^{N-2} \int_{D(0, \delta)} \frac{\sigma\left|x^{\prime}\right|^{2}+C(\sigma)\left|x^{\prime}\right|^{\frac{5}{2}}}{\left[\varepsilon^{2}+\left|x^{\prime}\right|^{2}\right]^{N-1}} d x^{\prime} \\
\leq & C \varepsilon\left(\sigma+C(\sigma) \varepsilon^{\frac{1}{2}}\right)
\end{aligned}
$$

since $N \geq 4$. Moreover, since $\sigma$ is arbitrary, we have

$$
\int_{D(0, \delta)} d x^{\prime} \int_{g\left(x^{\prime}\right)}^{h\left(x^{\prime}\right)} N^{\frac{N-2}{2}}(N-2)^{\frac{N+2}{2}} \varepsilon^{N-2} \frac{|x|^{2}}{\left[\varepsilon^{2}+|x|^{2}\right]^{N}} d x_{N}=o(\varepsilon) .
$$

Finally, we deduce from equation (3.10) and equation (3.11) that

$$
\int_{\Omega}\left|\nabla u_{\varepsilon}\right|^{2} d x=\int_{\mathbb{R}_{+}^{N}}\left|\nabla u_{\varepsilon}\right|^{2} d x-N^{\frac{N-2}{2}}(N-2)^{\frac{N+2}{2}} \varepsilon \int_{\mathbb{R}^{N-1}} \frac{\left|x^{\prime}\right|^{2} g\left(x^{\prime}\right)}{\left[1+\left|x^{\prime}\right|^{2}\right]^{N}} d x^{\prime}+o(\varepsilon)
$$

which proves (i).

For (ii), we have

$$
\begin{aligned}
& \int_{\mathbb{R}_{+}^{N}} u_{\varepsilon}^{2^{*}} d x-\int_{\Omega} u_{\varepsilon}^{2^{*}} d x \\
& =\int_{\mathbb{R}^{N-1}} d x^{\prime} \int_{0}^{g\left(x^{\prime}\right)} N^{\frac{N}{2}}(N-2)^{\frac{N}{2}} \varepsilon^{N}\left[\varepsilon^{2}+|x|^{2}\right]^{-N} d x_{N} \\
& +\int_{D(0, \delta)} d x^{\prime} \int_{g\left(x^{\prime}\right)}^{h\left(x^{\prime}\right)} N^{\frac{N}{2}}(N-2)^{\frac{N}{2}} \varepsilon^{N}\left[\varepsilon^{2}+|x|^{2}\right]^{-N} d x_{N}+O\left(\varepsilon^{N}\right) .
\end{aligned}
$$


EXISTENCE RESULTS FOR SUPERLINEAR ELLIPTIC EQUATIONS WITH NONLINEAR BOUNDARY VALUE CONDITIO

A direct calculation implies

$$
\begin{aligned}
& \int_{D(0, \delta)} d x^{\prime} \int_{0}^{g\left(x^{\prime}\right)} N^{\frac{N}{2}}(N-2)^{\frac{N}{2}} \varepsilon^{N}\left[\varepsilon^{2}+|x|^{2}\right]^{-N} d x_{N} \\
& =\int_{\mathbb{R}^{N-1}} d x^{\prime} \int_{0}^{g\left(x^{\prime}\right)} N^{\frac{N}{2}}(N-2)^{\frac{N}{2}} \varepsilon^{N}\left[\varepsilon^{2}+|x|^{2}\right]^{-N} d x_{N}+O\left(\varepsilon^{N}\right) \\
& =N^{\frac{N}{2}}(N-2)^{\frac{N}{2}} \int_{\mathbb{R}^{N-1}} d x^{\prime} \int_{0}^{\varepsilon g\left(x^{\prime}\right)} \frac{1}{\left[1+|x|^{2}\right]^{N}} d x_{N}+o(\varepsilon) \\
& =N^{\frac{N}{2}}(N-2)^{\frac{N}{2}} \varepsilon \int_{\mathbb{R}^{N-1}} \frac{g\left(x^{\prime}\right)}{\left[1+\left|x^{\prime}\right|^{2}\right]^{N}} d x^{\prime}+o(\varepsilon) .
\end{aligned}
$$

Moreover, similar to equation (3.11), we have

$$
\int_{D(0, \delta)} d x^{\prime} \int_{g\left(x^{\prime}\right)}^{h\left(x^{\prime}\right)} N^{\frac{N}{2}}(N-2)^{\frac{N}{2}} \varepsilon^{N}\left[\varepsilon^{2}+|x|^{2}\right]^{-N} d x_{N}=o(\varepsilon) .
$$

Hence, it follows from equations (3.12), (3.13) and (3.14) that

$$
\int_{\Omega} u_{\varepsilon}^{\frac{2 N}{N-2}} d x=\int_{\mathbb{R}_{+}^{N}} u_{\varepsilon}^{\frac{2 N}{N-2}} d x-N^{\frac{N}{2}}(N-2)^{\frac{N}{2}} \varepsilon \int_{\mathbb{R}^{N-1}} \frac{g\left(x^{\prime}\right)}{\left[1+\left|x^{\prime}\right|^{2}\right]^{N}} d x^{\prime}+o(\varepsilon),
$$

which proves (ii).

As for (iii), we note that since $q \in\left(2,2_{*}\right)$, so we get that

$$
\begin{aligned}
\int_{\mathbb{R}^{N-1}} u_{\varepsilon}\left(x^{\prime}, 0\right)^{q} d x^{\prime} & =C \varepsilon^{\frac{N-2}{2} q} \int_{\mathbb{R}^{N-1}} \frac{1}{\left[\varepsilon^{2}+\left|x^{\prime}\right|^{2}\right]^{\frac{N-2}{2} q}} d x^{\prime} \\
& =C \varepsilon^{N-1-\frac{N-2}{2} q} \int_{\mathbb{R}^{N-1}} \frac{1}{\left[1+\left|x^{\prime}\right|^{2}\right]^{\frac{N-2}{2} q}} d x^{\prime} .
\end{aligned}
$$

Moreover, since $q \in\left(2, \frac{2(N-1)}{N-2}\right)$, we have $N-1-\frac{N-2}{2} q \in(0,1)$, hence we get

$$
\int_{\partial \Omega} u_{\varepsilon}^{q} d S=C \varepsilon^{N-1-\frac{N-2}{2} q}+o\left(\varepsilon^{(N-1)-\frac{N-2}{2} q}\right),
$$

which proves (iii).

(iv) is proved in [5].

With the above preparations, we can prove Theorem 1.2 with $N \geq 4$ now.

Proof of Theorem 1.2 with $N \geq 4$ : We use the Mountain Pass theorem to prove our result. First, we infer from Sobolev imbedding theorem, Sobolev trace inequality that

$$
I(u) \geq \frac{1}{2}\|u\|^{2}-C\|u\|^{2^{*}}-C\|u\|^{q},
$$

hence, there exist $\rho, \alpha>0$ such that

$$
I(u) \geq \alpha>0
$$


for $u \in \partial B_{\rho}$. Moreover, let $u_{0} \neq 0$ fixed, then it is easy to check that

$$
I\left(t u_{0}\right) \rightarrow-\infty
$$

as $t \rightarrow \infty$. So there exists $t_{0}>0$ such that $\left\|t_{0} u_{0}\right\|>\rho$ and

$$
I\left(t_{0} u_{0}\right)<0 \text {. }
$$

Define

$$
c=\inf _{\gamma \in \Gamma} \sup _{t \in[0,1]} I(\gamma(t))
$$

with

$$
\Gamma=\left\{\gamma \in C\left([0,1], H^{1}(\Omega)\right): \gamma(0)=0, \gamma(1)=t_{0} u_{0}\right\},
$$

then $c$ is a well-defined positive constant. In order to show that $c$ is indeed a critical value for $I$, we only need to show that

$$
c<\frac{1}{2 N} S^{\frac{N}{2}}
$$

For this purpose, we note that

$$
I\left(t u_{\varepsilon}\right)=\frac{t^{2}}{2}\left\|u_{\varepsilon}\right\|^{2}-\frac{t^{2^{*}}}{2^{*}} \int_{\Omega} u_{\varepsilon}^{2^{*}} d x-\frac{t^{q}}{q} \int_{\partial \Omega} u_{\varepsilon}^{q} d S .
$$

Hence there exists $t_{\varepsilon}>0$ such that $I\left(t_{\varepsilon} u_{\varepsilon}\right)$ attains its maximum at $t_{\varepsilon}$. Moreover, it follows from Lemma 3.2 that $t_{\varepsilon} \rightarrow 1$ as $\varepsilon \rightarrow 0$. Hence, for $N \geq 5$, we get

$$
\begin{aligned}
I\left(t_{\varepsilon} u_{\varepsilon}\right) & \leq \frac{t_{\varepsilon}^{2}}{2} \int_{\mathbb{R}_{+}^{N}}\left|\nabla u_{\varepsilon}\right|^{2} d x-\frac{t_{\varepsilon}^{2^{*}}}{2^{*}} \int_{\mathbb{R}_{+}^{N}}\left|u_{\varepsilon}\right|^{2^{*}} d x-\frac{t_{\varepsilon}^{2}}{2} N^{\frac{N-2}{2}}(N-2)^{\frac{N+2}{2}} \varepsilon \int_{\mathbb{R}^{N-1}} \frac{\left|x^{\prime}\right|^{2} g\left(x^{\prime}\right)}{\left[1+\left|x^{\prime}\right|^{2}\right]^{N}} d x^{\prime} \\
& +O\left(\varepsilon^{2}\right)-C \varepsilon^{N-1-\frac{N-2}{2} q}+C \varepsilon \int_{\mathbb{R}^{N-1}} \frac{g\left(x^{\prime}\right)}{\left[1+\left|x^{\prime}\right|^{2}\right]^{N}} d x^{\prime} .
\end{aligned}
$$

On the other hand, since the function

$$
f(t)=\frac{t^{2}}{2} \int_{\mathbb{R}_{+}^{N}}\left|\nabla u_{\varepsilon}\right|^{2} d x-\frac{t^{2^{*}}}{2^{*}} \int_{\mathbb{R}_{+}^{N}}\left|u_{\varepsilon}\right|^{2^{*}} d x
$$

attains its maximum at $t=1$ and the maximum is $\frac{1}{2 N} S^{\frac{N}{2}}$, hence we have

$$
\begin{aligned}
I\left(t_{\varepsilon} u_{\varepsilon}\right) & \leq \frac{1}{2 N} S^{\frac{N}{2}}-C \varepsilon \int_{\mathbb{R}^{N-1}} \frac{\left|x^{\prime}\right|^{2} g\left(x^{\prime}\right)}{\left[1+\left|x^{\prime}\right|^{2}\right]^{N}} d x^{\prime} \\
& +O\left(\varepsilon^{2}\right)-C \varepsilon^{N-1-\frac{N-2}{2} q}+C \varepsilon \int_{\mathbb{R}^{N-1}} \frac{g\left(x^{\prime}\right)}{\left[1+\left|x^{\prime}\right|^{2}\right]^{N}} d x^{\prime} .
\end{aligned}
$$

Since $q \in\left(2, \frac{2(N-1)}{N-2}\right)$, we get $N-1-\frac{N-2}{2} q \in(0,1)$. Finally, we infer from the above inequality that

for $\varepsilon$ small enough.

$$
I\left(t_{\varepsilon} u_{\varepsilon}\right)<\frac{1}{2 N} S^{\frac{N}{2}}
$$

The case $N=4$ is similar, we only need to replace $O\left(\varepsilon^{2}\right)$ by $O\left(\varepsilon^{2}|\ln \varepsilon|\right)$ in equation (3.17). The rest of the proof is the same as $N \geq 5$. 
Finally, we study the case $N=3$. In this case, we suppose the principal curvatures of $\partial \Omega$ at $x_{0} \in \partial \Omega$ belong to interval $(2 a, 2 A)$ for some $0<a \leq A<\infty$. Then we have $a\left|x^{\prime}\right|^{2} \leq h\left(x^{\prime}\right) \leq A\left|x^{\prime}\right|^{2}$ for $x^{\prime} \in D(0, \delta)$. With these notations, we have the following estimates.

Lemma 3.3. Suppose $N=3$ and $x_{0}$ as above, then we have the following estimates.

(i) $\int_{\Omega}\left|\nabla u_{\varepsilon}\right|^{2} d x \leq \int_{\mathbb{R}_{+}^{N}}\left|\nabla u_{\varepsilon}\right|^{2} d x-C \varepsilon|\ln \varepsilon|+O(\varepsilon)$;

(ii) $\int_{\Omega} u_{\varepsilon}^{6} d x \geq \int_{\mathbb{R}_{+}^{N}} u_{\varepsilon}^{6} d x-C \varepsilon$.

(iii) $\int_{\partial \Omega} u_{\varepsilon}^{q} d S \geq C \varepsilon^{2-\frac{q}{2}}$ for $q \in(2,4)$.

(iv) $\int_{\Omega} u_{\varepsilon}^{2} d x^{\prime}=O(\varepsilon)$.

Proof. As for (i), we infer from the above setting that

$$
\begin{aligned}
& \int_{\Omega}\left|\nabla u_{\varepsilon}\right|^{2} d x=\int_{\mathbb{R}_{+}^{N}}\left|\nabla u_{\varepsilon}\right|^{2} d x-\int_{D(0, \delta)} d x^{\prime} \int_{0}^{h\left(x^{\prime}\right)}\left|\nabla u_{\varepsilon}\right|^{2} d x_{N}+O(\varepsilon) \\
& \leq \int_{\mathbb{R}_{+}^{N}}\left|\nabla u_{\varepsilon}\right|^{2} d x-\int_{D(0, \delta)} d x^{\prime} \int_{0}^{a\left|x^{\prime}\right|^{2}}\left|\nabla u_{\varepsilon}\right|^{2} d x_{N}+O(\varepsilon) \\
& \leq \int_{\mathbb{R}_{+}^{N}}\left|\nabla u_{\varepsilon}\right|^{2} d x-C \varepsilon \int_{D(0, \delta)} \frac{a\left|x^{\prime}\right|^{4}}{\left(\varepsilon^{2}+\left|x^{\prime}\right|^{2}\right)^{N}} d x^{\prime}+O(\varepsilon) \\
& \leq \int_{\mathbb{R}_{+}^{N}}\left|\nabla u_{\varepsilon}\right|^{2} d x-C \varepsilon|\ln \varepsilon|+O(\varepsilon) .
\end{aligned}
$$

As for (ii), we have

$$
\begin{aligned}
\int_{\Omega} u_{\varepsilon}^{6} d x & =\int_{\mathbb{R}_{+}^{N}} u_{\varepsilon}^{6} d x-\int_{D(0, \delta)} d x^{\prime} \int_{0}^{h\left(x^{\prime}\right)} u_{\varepsilon}^{6} d x_{N}+O\left(\varepsilon^{3}\right) \\
& \geq \int_{\mathbb{R}_{+}^{N}} u_{\varepsilon}^{6} d x-\int_{D(0, \delta)} d x^{\prime} \int_{0}^{A\left|x^{\prime}\right|^{2}} u_{\varepsilon}^{6} d x_{N}+O\left(\varepsilon^{3}\right) \\
& \geq \int_{\mathbb{R}_{+}^{N}} u_{\varepsilon}^{6} d x-C \varepsilon \int_{\mathbb{R}^{N-1}} \frac{\left|x^{\prime}\right|^{2}}{\left[1+\left|x^{\prime}\right|^{2}\right]^{3}} d x^{\prime}+O\left(\varepsilon^{3}\right) \\
& \geq \int_{\mathbb{R}_{+}^{N}} u_{\varepsilon}^{6} d x-C \varepsilon .
\end{aligned}
$$

(iii) is the same as Lemma 3.2 and (iv) can be found in $[5$. This finishes the proof of this lemma.

Proof of Theorem 1.2 with $N=3$ : By the same reason as for $N \geq 4$, we conclude that the functional $I$ possesses the Mountain Pass structure, so we only need to show that the 
Mountain Pass level is below $\frac{1}{2 N} S^{\frac{N}{2}}$. For this purpose, we let $u_{\varepsilon}, t_{\varepsilon}$ the same as the case $N \geq 4$, then we have

$$
I\left(t_{\varepsilon} u_{\varepsilon}\right) \leq \frac{1}{2 N} S^{\frac{N}{2}}-C \varepsilon|\ln \varepsilon|+C \varepsilon-C \varepsilon^{2-\frac{q}{2}} .
$$

Since $q \in(2,4)$, we deduce that $2-\frac{q}{2} \in(0,1)$. Insert this into the above inequality, then we conclude that

$$
I\left(t_{\varepsilon} u_{\varepsilon}\right)<\frac{1}{2 N} S^{\frac{N}{2}}
$$

for $\varepsilon>0$ small enough. This finishes the proof of Theorem 1.2 for $N=3$.

\section{Proof of Theorem 1.3}

In this section, we study the existence result of another critical equation, i.e., the case $2<$ $r<\frac{2 N}{N-2}$ and $q=\frac{2(N-1)}{N-2}$. We first introduce the respective "limit equation" corresponding to equation (1.1) in this case. Consider the following equation

$$
\begin{cases}-\Delta u=0 & \text { in } \mathbb{R}_{+}^{N}, \\ \frac{\partial u}{\partial \nu}=|u|^{2_{*}-2} u & \text { on } \partial \mathbb{R}_{+}^{N},\end{cases}
$$

its ground state solutions are closely related to the following Sobolev trace inequality

$$
S_{T}=\inf _{u \in D^{1,2}\left(\mathbb{R}_{+}^{N}\right): u \neq 0} \frac{\int_{\mathbb{R}_{+}^{N}}|\nabla u|^{2} d x}{\left(\int_{\mathbb{R}^{N-1}}\left|u\left(x^{\prime}, 0\right)\right|^{2 *} d x^{\prime}\right)^{\frac{2}{2 *}}} .
$$

That is, the minimizers of $S_{T}$ multiplied by a constant are the solutions of equation (4.1). Moreover, the ground state solutions of equation (4.1) have the following form

$$
u_{\varepsilon}(x)=\frac{(N-2)^{\frac{N-2}{2}} \varepsilon^{\frac{N-2}{2}}}{\left[\left(\varepsilon+x_{N}\right)^{2}+\left|x^{\prime}\right|^{2}\right]^{\frac{N-2}{2}}} .
$$

With the above preparations, we can study the existence result of equation (1.1) in this case now. Obviously, the solutions of problem (1.1) in this situation correspond to the critical points of the functional

$$
I(u)=\frac{1}{2} \int_{\Omega}|\nabla u|^{2}+u^{2} d x-\frac{1}{r} \int_{\Omega}|u|^{r} d x-\frac{1}{2_{*}} \int_{\partial \Omega}|u|^{2 *} d S
$$

defined on $H^{1}(\Omega)$. First, we have the following compactness lemma.

Lemma 4.1. Under the assumptions of Theorem 1.3, the functional I satisfies the $(P S)_{c}$ condition for $c \in\left(0, \frac{1}{2(N-1)} S_{T}^{N-1}\right)$.

Proof. Let $\left\{u_{n}\right\} \subset H^{1}(\Omega)$ be a $(P S)_{c}$ sequence for $I$ with $c \in\left(0, \frac{1}{2(N-1)} S_{T}^{N-1}\right)$, that is,

$$
I\left(u_{n}\right) \rightarrow c
$$

and

$$
I^{\prime}\left(u_{n}\right) \rightarrow 0
$$


we will prove that $\left\{u_{n}\right\}$ has a convergent subsequence.

We first show that $\left\{u_{n}\right\}$ is bounded. For this purpose, we infer from the above two equalities that

$$
\frac{1}{2} \int_{\Omega}\left|\nabla u_{n}\right|^{2}+u_{n}^{2} d x-\frac{1}{r} \int_{\Omega}\left|u_{n}\right|^{r} d x-\frac{1}{2_{*}} \int_{\partial \Omega}\left|u_{n}\right|^{2 *} d S \rightarrow c
$$

and

$$
\int_{\Omega}\left|\nabla u_{n}\right|^{2}+u_{n}^{2} d x-\int_{\Omega}\left|u_{n}\right|^{r} d x-\int_{\partial \Omega}\left|u_{n}\right|^{2 *} d S=o(1)\left\|u_{n}\right\|
$$

If $r \leq \frac{2(N-1)}{N-2}$, then we deduce from the above two equations that

$$
\left(\frac{1}{2}-\frac{1}{r}\right)\left\|u_{n}\right\|^{2}+\left(\frac{1}{r}-\frac{1}{2_{*}}\right) \int_{\partial \Omega}\left|u_{n}\right|^{2 *} d S=c+o(1)+o(1)\left\|u_{n}\right\|,
$$

which implies that $\left\|u_{n}\right\|$ is bounded. Similarly, if $r>2_{*}$, then we can still get from equation (4.5) and equation (4.6) that

$$
\left(\frac{1}{2}-\frac{1}{2_{*}}\right)\left\|u_{n}\right\|^{2}+\left(\frac{1}{2_{*}}-\frac{1}{r}\right) \int_{\Omega}\left|u_{n}\right|^{r} d x=c+o(1)+o(1)\left\|u_{n}\right\|,
$$

which also implies that $\left\|u_{n}\right\|$ is bounded. So in both cases, we conclude that $\left\|u_{n}\right\|$ is bounded.

Next, we show that $\left\{u_{n}\right\}$ has a convergent subsequence. Since $\left\{u_{n}\right\}$ is bounded, we can suppose that, up to a subsequence, $u_{n} \rightarrow u$ in $H^{1}(\Omega), u_{n} \rightarrow u$ in $L^{r}(\Omega), u_{n} \rightarrow u$ in $L^{2}(\Omega)$ and $u_{n} \rightarrow u$ in $L^{2 *}(\partial \Omega)$. Now let $v_{n}=u_{n}-u$, then we deduce from Brezis-Lieb theorem [4] that

$$
\frac{1}{2} \int_{\Omega}\left|\nabla v_{n}\right|^{2} d x-\frac{1}{2_{*}} \int_{\partial \Omega}\left|v_{n}\right|^{2 *} d S=c-I(u)+o(1)
$$

and

$$
\int_{\Omega}\left|\nabla v_{n}\right|^{2} d x-\int_{\partial \Omega}\left|v_{n}\right|^{2 *} d S=o(1) .
$$

Hence, we get

$$
\int_{\Omega}\left|\nabla v_{n}\right|^{2} d x=\int_{\partial \Omega}\left|v_{n}\right|^{2 *} d S=2(N-1)(c-I(u))+o(1) .
$$

As before, to prove this lemma, it is equivalent to prove $v_{n} \rightarrow 0$ in $H^{1}(\Omega)$. We prove this conclusion by contradiction. Suppose on the contrary, that is, $v_{n} \not \rightarrow 0$ in $H^{1}(\Omega)$, then we will show that this will lead to a contradiction. In fact, we have

$$
\begin{aligned}
{[2(N-1)(c-I(u)+o(1))]^{\frac{2}{2 *}} } & =\left(\int_{\partial \Omega}\left(v_{n}\right)^{2 *} d S\right)^{\frac{2}{2 *}} \\
& \leq \frac{1}{S_{T}} \int_{\Omega}\left|\nabla v_{n}\right|^{2} d x \\
& =\frac{1}{S_{T}}[2(N-1)(c-I(u)+o(1))] .
\end{aligned}
$$


It follows from the above inequality that

$$
2(N-1)(c-I(u)+o(1)) \geq S_{T}^{N-1} .
$$

Let $n \rightarrow \infty$ in the above inequality and note that $I(u) \geq 0$, then we get that

$$
c \geq \frac{1}{2(N-1)} S^{N-1}
$$

which contradicts the choice of $c$. This finishes the proof of this lemma.

In order to prove the Mountain Pass level of functional $I$ is indeed below $\frac{1}{2(N-1)} S^{N-1}$, we need to estimate the Mountain Pass value carefully. As before, we assume that the ball $B_{R}(\bar{x})$ contains $\Omega$ and $\partial B_{R}(\bar{x}) \cap \bar{\Omega} \neq \emptyset$. Suppose that $x_{0} \in \partial B_{R}(\bar{x}) \cap \bar{\Omega}$, then we have $2 \alpha_{i} \geq \frac{1}{R}$ for each $1 \leq i \leq N-1$, where $2 \alpha_{i}(i=1, \cdots, N-1)$ are the principal curvatures of $\partial \Omega$ at $x_{0}$. We can suppose $x_{0}=0$ and $\Omega \subset\left\{x: x_{N}>0\right\}$ without loss of generality, then the boundary of $\Omega$ can be represented by

$$
x_{N}=h\left(x^{\prime}\right)=\sum_{i=1}^{N-1} \alpha_{i} x_{i}^{2}+o\left(\left|x^{\prime}\right|^{2}\right), \quad \forall x^{\prime}=\left(x_{1}, x_{2}, \cdots, x_{N-1}\right) \in D(0, \delta)
$$

for some $\delta>0$, where we denote $D(0, \delta)=B_{\delta}(0) \cap\left\{x_{N}=0\right\}$. In the following, we denote $g\left(x^{\prime}\right)=\sum_{i=1}^{N-1} \alpha_{i} x_{i}^{2}$ for simplicity.

Lemma 4.2. Let $N \geq 4$ and $u_{\varepsilon}$ be defined in equation (4.3), then we have the following estimates.

(i) $\int_{\Omega}\left|\nabla u_{\varepsilon}\right|^{2} d x=\int_{\mathbb{R}_{+}^{N}}\left|\nabla u_{\varepsilon}\right|^{2} d x-(N-2)^{N} \varepsilon \int_{\mathbb{R}^{N-1}} \frac{g\left(x^{\prime}\right)}{\left[1+\left|x^{\prime}\right|^{2}\right]^{N-1}} d x^{\prime}+o(\varepsilon)$;

(ii) $\int_{\partial \Omega} u_{\varepsilon}^{2 *} d S=\int_{\mathbb{R}^{N-1}} u_{\varepsilon}\left(x^{\prime}, 0\right)^{2 *} d x^{\prime}-2(N-1)(N-2)^{N-1} \varepsilon \int_{\mathbb{R}^{N-1}} \frac{g\left(x^{\prime}\right)}{\left[1+\left|x^{\prime}\right|\right]^{N}} d x^{\prime}+o(\varepsilon)$;

(iii) $\int_{\Omega} u_{\varepsilon}^{2} d x= \begin{cases}O\left(\varepsilon^{2}|\ln \varepsilon|\right) & \text { if } N=4, \\ O\left(\varepsilon^{2}\right) & \text { if } N \geq 5 .\end{cases}$ 
EXISTENCE RESULTS FOR SUPERLINEAR ELLIPTIC EQUATIONS WITH NONLINEAR BOUNDARY VALUE CONDITIO

Proof. For (i), a direct calculation implies that

$$
\begin{aligned}
& \int_{\mathbb{R}_{+}^{N}}\left|\nabla u_{\varepsilon}\right|^{2} d x-\int_{\Omega}\left|\nabla u_{\varepsilon}\right|^{2} d x \\
& =\int_{\mathbb{R}^{N-1}} d x^{\prime} \int_{0}^{g\left(x^{\prime}\right)}(N-2)^{N} \varepsilon^{N-2} \frac{1}{\left[\left(\varepsilon+x_{N}\right)^{2}+\left|x^{\prime}\right|^{2}\right]^{N-1}} d x_{N} \\
& +\int_{D(0, \delta)} d x^{\prime} \int_{g\left(x^{\prime}\right)}^{h\left(x^{\prime}\right)}(N-2)^{N} \varepsilon^{N-2} \frac{1}{\left[\left(\varepsilon+x_{N}\right)^{2}+\left|x^{\prime}\right|^{2}\right]^{N-1}} d x_{N}+O\left(\varepsilon^{N-2}\right) \\
& =\int_{\mathbb{R}^{N-1}} d x^{\prime} \int_{0}^{\varepsilon g\left(x^{\prime}\right)}(N-2)^{N} \frac{1}{\left[\left(1+x_{N}\right)^{2}+\left|x^{\prime}\right|^{2}\right]^{N-1}} d x_{N} \\
& +\int_{D(0, \delta)} d x^{\prime} \int_{g\left(x^{\prime}\right)}^{h\left(x^{\prime}\right)}(N-2)^{N} \varepsilon^{N-2} \frac{1}{\left[\left(\varepsilon+x_{N}\right)^{2}+\left|x^{\prime}\right|^{2}\right]^{N-1}} d x_{N}+O\left(\varepsilon^{N-2}\right) \\
& =(N-2)^{N} \varepsilon \int_{\mathbb{R}^{N-1}}^{h\left(1+\left|x^{\prime}\right|^{2}\right]^{N-1}} d x^{\prime} \\
& +\int_{D(0, \delta)} d x^{\prime} \int_{g\left(x^{\prime}\right)}^{h\left(x^{\prime}\right)}(N-2)^{N} \varepsilon^{N-2} \frac{1}{\left[\left(\varepsilon+x_{N}\right)^{2}+\left|x^{\prime}\right|^{2}\right]^{N-1}} d x_{N}+o(\varepsilon) .
\end{aligned}
$$

On the other hand, since $h\left(x^{\prime}\right)=g\left(x^{\prime}\right)+o\left(\left|x^{\prime}\right|^{2}\right)$, it follows that $\forall \sigma>0$, there exists $C(\sigma)>0$ such that $\left|h\left(x^{\prime}\right)-g\left(x^{\prime}\right)\right| \leq \sigma\left|x^{\prime}\right|^{2}+C(\sigma)\left|x^{\prime}\right|^{\frac{5}{2}}$ for $x^{\prime} \in D(0, \delta)$. Therefore, we have

$$
\begin{aligned}
& \int_{D(0, \delta)} d x^{\prime} \int_{g\left(x^{\prime}\right)}^{h\left(x^{\prime}\right)}(N-2)^{N} \varepsilon^{N-2} \frac{1}{\left[\left(\varepsilon+x_{N}\right)^{2}+|x|^{2}\right]^{N-1}} d x_{N} \\
\leq & C \varepsilon^{N-2} \int_{D(0, \delta)} d x^{\prime} \int_{g\left(x^{\prime}\right)}^{h\left(x^{\prime}\right)} \frac{1}{\left[\left(\varepsilon+x_{N}\right)^{2}+|x|^{2}\right]^{N-1}} d x_{N} \\
\leq & C \varepsilon^{N-2} \int_{D(0, \delta)} \frac{\left|h\left(x^{\prime}\right)-g\left(x^{\prime}\right)\right|}{\left[\varepsilon^{2}+\left|x^{\prime}\right|^{2}\right]^{N-1}} d x^{\prime} \\
\leq & C \varepsilon^{N-2} \int_{D(0, \delta)} \frac{\sigma\left|x^{\prime}\right|^{2}+C(\sigma)\left|x^{\prime}\right|^{\frac{5}{2}}}{\left[\varepsilon^{2}+\left|x^{\prime}\right|^{2}\right]^{N-1}} d x^{\prime} \\
\leq & C \varepsilon\left(\sigma+C(\sigma) \varepsilon^{\frac{1}{2}}\right) .
\end{aligned}
$$

Since $\sigma$ is arbitrary, we have

$$
\int_{D(0, \delta)} d x^{\prime} \int_{g\left(x^{\prime}\right)}^{h\left(x^{\prime}\right)}(N-2)^{N} \varepsilon^{N-2} \frac{1}{\left[\left(\varepsilon+x_{N}\right)^{2}+\left|x^{\prime}\right|^{2}\right]^{N-1}} d x_{N}=o(\varepsilon) .
$$

Finally, we deduce from equation (4.11) and equation (4.12) that

$$
\int_{\Omega}\left|\nabla u_{\varepsilon}\right|^{2} d x=\int_{\mathbb{R}_{+}^{N}}\left|\nabla u_{\varepsilon}\right|^{2} d x-(N-2)^{N} \varepsilon \int_{\mathbb{R}^{N-1}} \frac{g\left(x^{\prime}\right)}{\left[1+\left|x^{\prime}\right|^{2}\right]^{N-1}} d x^{\prime}+o(\varepsilon),
$$

this proves (i). 
As for (ii), if we denote

$$
f(r)=u_{\varepsilon}\left(x^{\prime}, r g\left(x^{\prime}\right)\right)^{\frac{2(N-1)}{N-2}} \sqrt{1+r^{2}\left|\nabla g\left(x^{\prime}\right)\right|^{2}},
$$

then we have

$$
\begin{aligned}
& \int_{\partial \Omega} u_{\varepsilon}^{2 *} d S-\int_{\mathbb{R}^{N-1}} u_{\varepsilon}\left(x^{\prime}, 0\right)^{2 *} d x^{\prime} \\
& =\int_{\mathbb{R}^{N-1}}[f(1)-f(0)] d x^{\prime}+o(\varepsilon) \\
& =\int_{\mathbb{R}^{N-1}} f^{\prime}\left(r_{\varepsilon}\right) d x^{\prime}+o(\varepsilon) \\
& =-\int_{\mathbb{R}^{N-1}} 2(N-1)(N-2)^{N-1} \varepsilon^{N-1} \frac{\left(\varepsilon+r_{\varepsilon} g\left(x^{\prime}\right)\right) g\left(x^{\prime}\right)}{\left[\left(\varepsilon+r_{\varepsilon} g\left(x^{\prime}\right)\right)^{2}+\left|x^{\prime}\right|^{2}\right]^{N}} \sqrt{1+r_{\varepsilon}^{2}\left|\nabla g\left(x^{\prime}\right)\right|^{2}} d x^{\prime}+o(\varepsilon) \\
& =-2(N-1)(N-2)^{N-1} \varepsilon \int_{\mathbb{R}^{N-1}} \frac{g\left(x^{\prime}\right)}{\left[1+\left|x^{\prime}\right|^{2}\right]^{N}} d x^{\prime}+o(\varepsilon),
\end{aligned}
$$

which proves (iii)

(iii) can be proved as in [5].

With the above preparations, we can prove Theorem 1.3 for $N \geq 4$ now.

Proof of Theorem 1.3 with $N \geq 4$ : We use the Mountain Pass theorem to prove our result. First, we note that

$$
I(u) \geq \frac{1}{2}\|u\|^{2}-C\|u\|^{r}-C\|u\|^{2 *},
$$

hence, there exist $\rho, \alpha>0$ such that

$$
I(u) \geq \alpha>0
$$

for $u \in \partial B_{\rho}$. Moreover, let $u_{0} \in H^{1}(\Omega)$ and $u_{0} \neq 0$ fixed, then it is easy to check that

$$
I\left(t u_{0}\right) \rightarrow-\infty
$$

as $t \rightarrow \infty$. So there exists $t_{0}>0$ such that $\left\|t_{0} u_{0}\right\|>\rho$ and

$$
I\left(t_{0} u_{0}\right)<0 \text {. }
$$

Define

$$
c=\inf _{\gamma \in \Gamma} \sup _{t \in[0,1]} I(\gamma(t))
$$

with

$$
\Gamma=\left\{\gamma \in C\left([0,1], H^{1}(\Omega)\right): \gamma(0)=0, \gamma(1)=t_{0} u_{0}\right\}
$$

then $c$ is a well-defined positive constant. In order to show that $c$ is indeed a critical value for $I$, we only need to show that

$$
c<\frac{1}{2(N-1)} S_{T}^{N-1} .
$$


EXISTENCE RESULTS FOR SUPERLINEAR ELLIPTIC EQUATIONS WITH NONLINEAR BOUNDARY VALUE CONDITIO

For this purpose, we note that

$$
I\left(t u_{\varepsilon}\right)=\frac{t^{2}}{2}\left\|u_{\varepsilon}\right\|^{2}-\frac{t^{r}}{r} \int_{\Omega} u_{\varepsilon}^{r} d x-\frac{t^{2 *}}{2_{*}} \int_{\partial \Omega} u_{\varepsilon}^{2_{*}} d S .
$$

Hence there exists $t_{\varepsilon}$ such that $I\left(t_{\varepsilon} u_{\varepsilon}\right)$ attains its maximum at $t_{\varepsilon}$. Moreover, it follows from Lemma 4.2 that $t_{\varepsilon} \rightarrow 1$ as $\varepsilon \rightarrow 0$. Hence, for $N \geq 5$, we get

$$
\begin{aligned}
I\left(t_{\varepsilon} u_{\varepsilon}\right) & \leq \frac{t_{\varepsilon}^{2}}{2} \int_{\mathbb{R}_{+}^{N}}\left|\nabla u_{\varepsilon}\right|^{2} d x-\frac{t_{\varepsilon}^{2 *}}{2_{*}} \int_{\mathbb{R}^{N-1}}\left|u_{\varepsilon}\left(x^{\prime}, 0\right)\right|^{2 *} d x^{\prime}-\frac{t_{\varepsilon}^{2}}{2}(N-2)^{N} \varepsilon \int_{\mathbb{R}^{N-1}} \frac{g\left(x^{\prime}\right)}{\left[1+\left|x^{\prime}\right|^{2}\right]^{N-1}} d x^{\prime} \\
& -\frac{t_{\varepsilon}^{r}}{r} \int_{\Omega} u_{\varepsilon}^{r} d x+t_{\varepsilon}^{2 *}(N-2)^{N} \varepsilon \int_{\mathbb{R}^{N-1}} \frac{g\left(x^{\prime}\right)}{\left[1+\left|x^{\prime}\right|^{2}\right]^{N}} d x^{\prime}+O\left(\varepsilon^{2}\right) .
\end{aligned}
$$

We note that the function

$$
f(t)=\frac{t^{2}}{2} \int_{\mathbb{R}_{+}^{N}}\left|\nabla u_{\varepsilon}\right|^{2} d x-\frac{t^{2 *}}{2^{*}} \int_{\mathbb{R}^{N-1}}\left|u_{\varepsilon}\left(x^{\prime}, 0\right)\right|^{2 *} d x^{\prime}
$$

attains its maximum at $t=1$ and the maximum is $\frac{1}{2(N-1)} S_{T}^{N-1}$. Hence, to prove $I\left(t_{\varepsilon} u_{\varepsilon}\right)<0$ for $\varepsilon$ small enough, it is sufficient to prove

$$
(N-2)^{N} \int_{\mathbb{R}^{N-1}} \frac{g\left(x^{\prime}\right)}{\left[1+\left|x^{\prime}\right|^{2}\right]^{N}} d x^{\prime}-\frac{1}{2}(N-2)^{N} \int_{\mathbb{R}^{N-1}} \frac{g\left(x^{\prime}\right)}{\left[1+\left|x^{\prime}\right|^{2}\right]^{N-1}} d x^{\prime}<0 .
$$

In the following, we will show that equation (4.15) indeed holds.

A direct calculation shows that

$$
\begin{aligned}
& \int_{\mathbb{R}^{N-1}} \frac{g\left(x^{\prime}\right)}{\left[1+\left|x^{\prime}\right|^{2}\right]^{N-1}} d x^{\prime} \\
& =\int_{\mathbb{R}^{N-1}} \frac{\sum_{i=1}^{N-1} \alpha_{i} x_{i}^{2}}{\left[1+\left|x^{\prime}\right|^{2}\right]^{N-1}} d x^{\prime} \\
& =\frac{1}{2} H(0) \int_{\mathbb{R}^{N-1}} \frac{\left|x^{\prime}\right|^{2}}{\left[1+\left|x^{\prime}\right|^{2}\right]^{N-1}} d x^{\prime} \\
& =\frac{1}{2} H(0) \omega_{N-2} \int_{0}^{\infty} \frac{r^{N}}{\left[1+r^{2}\right]^{N-1}} d r
\end{aligned}
$$

where $H(0)=\frac{2}{N-1} \sum_{i=1}^{N-1} \alpha_{i}$ is the mean curvature of $\partial \Omega$ at $0, \omega_{N-2}$ is the area of the unit sphere in $R^{N-1}$. Similarly, we have

$$
\int_{\mathbb{R}^{N-1}} \frac{g\left(x^{\prime}\right)}{\left[1+\left|x^{\prime}\right|^{2}\right]^{N}} d x^{\prime}=\frac{1}{2} H(0) \omega_{N-2} \int_{0}^{\infty} \frac{r^{N}}{\left[1+r^{2}\right]^{N}} d r .
$$


On the other hand, we note that

$$
\begin{aligned}
& \int_{0}^{\infty} \frac{r^{N}}{\left[1+r^{2}\right]^{N-1}} d r \\
& =\frac{2(N-1)}{N+1} \int_{0}^{\infty} \frac{r^{N+2}}{\left[1+r^{2}\right]^{N}} d r \\
& =\frac{2(N-1)}{N+1}\left[\int_{0}^{\infty} \frac{\left(1+r^{2}\right) r^{N}}{\left[1+r^{2}\right]^{N}} d r-\int_{0}^{\infty} \frac{r^{N}}{\left[1+r^{2}\right]^{N}} d r\right] \\
& =\frac{2(N-1)}{N+1}\left[\int_{0}^{\infty} \frac{r^{N}}{\left[1+r^{2}\right]^{N-1}} d r-\int_{0}^{\infty} \frac{r^{N}}{\left[1+r^{2}\right]^{N}} d r\right]
\end{aligned}
$$

So we get

$$
\int_{0}^{\infty} \frac{r^{N}}{\left[1+r^{2}\right]^{N-1}} d r=\frac{2(N-1)}{N-3} \int_{0}^{\infty} \frac{r^{N}}{\left[1+r^{2}\right]^{N}} d r
$$

We infer from the above equations that

$$
\begin{aligned}
& (N-2)^{N} \int_{\mathbb{R}^{N-1}} \frac{g\left(x^{\prime}\right)}{\left[1+\left|x^{\prime}\right|^{2}\right]^{N}} d x^{\prime}-\frac{1}{2}(N-2)^{N} \int_{\mathbb{R}^{N-1}} \frac{g\left(x^{\prime}\right)}{\left[1+\left|x^{\prime}\right|^{2}\right]^{N-1}} d x^{\prime} \\
& =-(N-2)^{N} H(0) \omega_{N-2} \frac{1}{N-3} \int_{0}^{\infty} \frac{r^{N}}{\left[1+r^{2}\right]^{N}} d r<0
\end{aligned}
$$

provided $H(0)>0$. This proved the case $N \geq 5$.

The case $N=4$ is similar. We only need to replace $O\left(\varepsilon^{2}\right)$ by $O\left(\varepsilon^{2}|\ln \varepsilon|\right)$ in equation (4.14), the rest of the proof is the same as $N \geq 5$. We omit the details.

Finally, we study the case $N=3$. In this case, we suppose the principal curvatures of $\partial \Omega$ at $x_{0} \in \partial \Omega$ belong to interval $(2 a, 2 A)$ for some $0<a \leq A<\infty$. Then we have $a\left|x^{\prime}\right|^{2} \leq h\left(x^{\prime}\right) \leq A\left|x^{\prime}\right|^{2}$ for $x^{\prime} \in D(0, \delta)$. Moreover, we have the following estimates.

Lemma 4.3. Suppose $N=3$ and $x_{0}$ as above, then we have the following estimates.

(i) $\int_{\Omega}\left|\nabla u_{\varepsilon}\right|^{2} d x \leq \int_{\mathbb{R}_{+}^{N}}\left|\nabla u_{\varepsilon}\right|^{2} d x-C \varepsilon|\ln \varepsilon|+O(\varepsilon)$;

(ii) $\int_{\partial \Omega} u_{\varepsilon}^{4} d S=\int_{\mathbb{R}^{2}} u_{\varepsilon}\left(x^{\prime}, 0\right)^{4} d x^{\prime}-C \varepsilon+o(\varepsilon)$.

(iii) $\int_{\Omega} u_{\varepsilon}^{2} d x^{\prime}=O(\varepsilon)$. 
EXISTENCE RESULTS FOR SUPERLINEAR ELLIPTIC EQUATIONS WITH NONLINEAR BOUNDARY VALUE CONDITIO

Proof. As for (i), we infer from the above setting that

$$
\begin{aligned}
& \int_{\Omega}\left|\nabla u_{\varepsilon}\right|^{2} d x=\int_{\mathbb{R}_{+}^{N}}\left|\nabla u_{\varepsilon}\right|^{2} d x-\int_{D(0, \delta)} d x^{\prime} \int_{0}^{h\left(x^{\prime}\right)}\left|\nabla u_{\varepsilon}\right|^{2} d x_{N}+O(\varepsilon) \\
& \leq \int_{\mathbb{R}_{+}^{N}}\left|\nabla u_{\varepsilon}\right|^{2} d x-C \int_{D(0, \delta)} d x^{\prime} \int_{0}^{a\left|x^{\prime}\right|^{2}} \frac{\varepsilon}{\left[\left(\varepsilon+x_{N}\right)^{2}+\left|x^{\prime}\right|^{2}\right]^{2}} d x_{N}+O(\varepsilon) \\
& \leq \int_{\mathbb{R}_{+}^{N}}\left|\nabla u_{\varepsilon}\right|^{2} d x-C \int_{D\left(0, \varepsilon^{-1} \delta\right)} d x^{\prime} \int_{0}^{\varepsilon a\left|x^{\prime}\right|^{2}} \frac{1}{\left[\left(1+x_{N}\right)^{2}+\left|x^{\prime}\right|^{2}\right]^{2}} d x_{N}+O(\varepsilon) \\
& =\int_{\mathbb{R}_{+}^{N}}\left|\nabla u_{\varepsilon}\right|^{2} d x-C \varepsilon \int_{D\left(0, \varepsilon^{-1} \delta\right)} \frac{a\left|x^{\prime}\right|^{2}}{\left[1+\left|x^{\prime}\right|^{2}\right]^{2}} d x^{\prime}+O(\varepsilon) \\
& =\int_{\mathbb{R}_{+}^{N}}\left|\nabla u_{\varepsilon}\right|^{2} d x-C \varepsilon[C+|\ln \varepsilon|]+O(\varepsilon) \\
& =\int_{\mathbb{R}_{+}^{N}}\left|\nabla u_{\varepsilon}\right|^{2} d x-C \varepsilon|\ln \varepsilon|+O(\varepsilon),
\end{aligned}
$$

this proves (i).

(ii) is the same as $N \geq 4$, we omit the details.

(iii) can be proved in a similar way as in [5]. In fact, we have

$$
\begin{aligned}
\int_{\Omega} u_{\varepsilon}^{2} d x & \leq C \int_{B_{R}} \frac{\varepsilon}{\left(\varepsilon+x_{N}\right)^{2}+\left|x^{\prime}\right|^{2}} d x \\
& \leq C \varepsilon \int_{B_{R}} \frac{1}{\varepsilon^{2}+|x|^{2}} d x \\
& =C \varepsilon \int_{0}^{R} \frac{r^{2}}{\varepsilon^{2}+r^{2}} d r \\
& =C \varepsilon\left[\int_{0}^{\varepsilon} \frac{1}{2} d r+\int_{\varepsilon}^{R} 1 d r\right] \\
& =C \varepsilon\left[\frac{1}{2} \varepsilon+(R-\varepsilon)\right] \\
& \leq C \varepsilon .
\end{aligned}
$$

This finishes the proof of this lemma.

Proof of Theorem 1.3 with $N=3$ : By the same reason as for $N \geq 4$, we conclude that the functional $I$ possesses the Mountain Pass structure, so we only need to show that the Mountain Pass level is below $\frac{1}{2(N-1)} S_{T}^{N-1}$. For this purpose, we let $u_{\varepsilon}, t_{\varepsilon}$ the same as the case $N \geq 4$, then we have

$$
I\left(t_{\varepsilon} u_{\varepsilon}\right) \leq \frac{1}{2(N-1)} S_{T}^{N-1}-C \varepsilon|\ln \varepsilon|-C \int_{\Omega} u_{\varepsilon}^{r} d r+O(\varepsilon) .
$$


It is easy to conclude from the above inequality that

$$
I\left(t_{\varepsilon} u_{\varepsilon}\right)<\frac{1}{2(N-1)} S_{T}^{N-1}
$$

for $\varepsilon>0$ small enough. This finishes the proof of Theorem 1.3 for $N=3$.

\section{Proof of Theorem 1.4}

In the finally section, we study the existence result of equation (1.1) with double critical exponents, i.e., $r=2^{*}$ and $q=2_{*}$. We first consider the following limit problem

$$
\begin{cases}-\Delta u=u^{2^{*}-1} & \text { in } \quad \mathbb{R}_{+}^{N} \\ \frac{\partial u}{\partial \nu}=u^{2_{*}-1} & \text { on } \quad \partial \mathbb{R}_{+}^{N}\end{cases}
$$

Y.Li and M.Zhu [19] classified all the positive solutions of problem (5.1). More precisely, they proved the positive solutions have the following form

$$
u_{\varepsilon}(x)=\frac{[N(N-2)]^{\frac{N-2}{4}} \varepsilon^{\frac{N-2}{2}}}{\left[\varepsilon^{2}+\left|x^{\prime}\right|^{2}+\left(x_{N}+\varepsilon x_{N}^{0}\right)^{2}\right]^{\frac{N-2}{2}}},
$$

where $x_{N}^{0}=\left(\frac{N}{N-2}\right)^{\frac{1}{2}}$. Let

$$
I_{\infty}(u)=\frac{1}{2} \int_{\mathbb{R}_{+}^{N}}|\nabla u|^{2} d x-\frac{1}{2^{*}} \int_{\mathbb{R}_{+}^{N}}|u|^{2^{*}} d x-\frac{1}{2_{*}} \int_{\partial \mathbb{R}_{+}^{N}}|u(x, 0)|^{2 *} d x^{\prime}
$$

defined on $D^{1,2}\left(\mathbb{R}_{+}^{N}\right), c_{\infty}=I_{\infty}\left(u_{\varepsilon}\right)$ and

$$
I(u)=\frac{1}{2} \int_{\Omega}|\nabla u|^{2}+u^{2} d x-\frac{1}{2^{*}} \int_{\Omega}|u|^{2^{*}} d x-\frac{1}{2_{*}} \int_{\partial \Omega}|u|^{2 *} d S
$$

defined on $H^{1}(\Omega)$, then we have the following local compactness result.

Lemma 5.1. Let $\left\{u_{n}\right\}$ be a $(P S)_{c}$ sequence for $I$ with $c \in\left(0, c_{\infty}\right)$, that is, $I\left(u_{n}\right) \rightarrow c$ and $I^{\prime}\left(u_{n}\right) \rightarrow 0$ as $n \rightarrow \infty$, then $\left\{u_{n}\right\}$ has a convergent subsequence.

Proof. Let $\left\{u_{n}\right\}$ be a $(P S)_{c}$ sequence for $I$ with $c \in\left(0, c_{\infty}\right)$, that is,

$$
I\left(u_{n}\right) \rightarrow c
$$

and

$$
I^{\prime}\left(u_{n}\right) \rightarrow 0
$$

as $n \rightarrow \infty$, we will show that $\left\{u_{n}\right\}$ has a convergent subsequence.

We first show that $\left\{u_{n}\right\}$ is bounded. By means of a $(P S)_{c}$ sequence, we have the following two equations

$$
I\left(u_{n}\right)=\frac{1}{2} \int_{\Omega}\left|\nabla u_{n}\right|+u_{n}^{2} d x-\frac{1}{2^{*}} \int_{\Omega}\left|u_{n}\right|^{2^{*}} d x-\frac{1}{2_{*}} \int_{\partial \Omega}\left|u_{n}\right|^{2_{*}} d S=c+o(1)
$$

and

$$
\left\langle I^{\prime}\left(u_{n}\right), u_{n}\right\rangle=\int_{\Omega}\left|\nabla u_{n}\right|+u_{n}^{2} d x-\int_{\Omega}\left|u_{n}\right|^{2^{*}} d x-\int_{\partial \Omega}\left|u_{n}\right|^{2_{*}} d S=o(1)\left\|u_{n}\right\| .
$$


We infer from the above two equations that

$$
\left(\frac{1}{2}-\frac{1}{2_{*}}\right)\left\|u_{n}\right\|^{2} \leq I\left(u_{n}\right)-\frac{1}{2_{*}}\left\langle I^{\prime}\left(u_{n}\right), u_{n}\right\rangle=c+o(1)+o(1)\left\|u_{n}\right\| .
$$

Hence, we conclude from the above equation that $\left\{u_{n}\right\}$ is bounded.

Next, we show that $\left\{u_{n}\right\}$ has a convergent subsequence. Since $u_{n}$ is bounded, we can suppose that $u_{n} \rightarrow u_{0}$ in $H^{1}(\Omega), u_{n} \rightarrow u_{0}$ in $L^{2^{*}}(\Omega), u_{n} \rightarrow u_{0}$ in $L^{2 *}(\partial \Omega)$ and $u_{n} \rightarrow u_{0}$ in $L^{2}(\Omega)$. Let $v_{n}=u_{n}-u_{0}$, if $v_{n} \rightarrow 0$ in $H^{1}(\Omega)$, then the proof is complete. So in the following, we assume that $v_{n} \not \rightarrow 0$. We deduce from Brezis-Lieb Theorem [4] that

$$
\frac{1}{2} \int_{\Omega}\left|\nabla v_{n}\right| d x-\frac{1}{2^{*}} \int_{\Omega}\left|v_{n}\right|^{2^{*}} d x-\frac{1}{2_{*}} \int_{\partial \Omega}\left|v_{n}\right|^{2 *} d S=c+o(1)-I\left(u_{0}\right)
$$

and

$$
\int_{\Omega}\left|\nabla v_{n}\right| d x-\int_{\Omega}\left|v_{n}\right|^{2^{*}} d x-\int_{\partial \Omega}\left|v_{n}\right|^{2_{*}} d S=o(1) .
$$

We distinguishes two cases:

Case 1: $\int_{\partial \Omega}\left|v_{n}\right|^{2 *} d S \nrightarrow 0$.

Case 2: $\int_{\partial \Omega}\left|v_{n}\right|^{2 *} d S \rightarrow 0$ but $\int_{\Omega}\left|v_{n}\right|^{2^{*}} d x \not \rightarrow 0$.

If case 1 occurs, then we conclude that $\left\{v_{n}\right\}$ is a $(P S)$ sequence for $I_{\infty}$, hence we have that

$$
\lim \inf _{n \rightarrow \infty} I\left(v_{n}\right) \geq c_{\infty}
$$

which further implies

$$
\lim \inf _{n \rightarrow \infty} I\left(u_{n}\right)=\lim \inf _{n \rightarrow \infty} I\left(v_{n}\right)+I\left(u_{0}\right) \geq \lim \inf _{n \rightarrow \infty} I\left(v_{n}\right) \geq c_{\infty}
$$

which contradicts that $c \in\left(0, c_{\infty}\right)$.

Similarly, if case 2 occurs, then we conclude that $\left\{v_{n}\right\}$ is a $(P S)$ sequence for $\bar{I}$ or $\tilde{I}$ which are defined by

$$
\bar{I}(u)=\frac{1}{2} \int_{\mathbb{R}_{+}^{N}}|\nabla u|^{2} d x-\frac{1}{2^{*}} \int_{\mathbb{R}_{+}^{N}}\left|u_{n}\right|^{2^{*}} d x, \quad u \in D^{1,2}\left(\mathbb{R}_{+}^{N}\right)
$$

and

$$
\tilde{I}(u)=\frac{1}{2} \int_{\mathbb{R}^{N}}|\nabla u|^{2} d x-\frac{1}{2^{*}} \int_{\mathbb{R}^{N}}\left|u_{n}\right|^{2^{*}} d x, \quad u \in D^{1,2}\left(\mathbb{R}^{N}\right)
$$

respectively. We note that the ground state levels of $\bar{I}$ and $\tilde{I}$ are $\frac{1}{2 N} S^{\frac{N}{2}}$ and $\frac{1}{N} S^{\frac{N}{2}}$ respectively. Moreover, a easy calculation shows that $\frac{1}{2 N} S^{\frac{N}{2}}>c_{\infty}$. So we deduce that

$$
\lim \inf _{n \rightarrow \infty} I\left(u_{n}\right) \geq \lim \inf _{n \rightarrow \infty} I\left(v_{n}\right)+I\left(u_{0}\right) \geq \lim \inf _{n \rightarrow \infty} I\left(v_{n}\right)>c_{\infty}
$$

which contradicts that $c \in\left(0, c_{\infty}\right)$.

Finally, we must have $v_{n} \rightarrow 0$ or $u_{n} \rightarrow u_{0}$ in $H^{1}(\Omega)$. This completes the proof of this lemma. 
In order to imply the Mountain Pass theorem to functional $I$, we must show that the Mountain Pass level of $I$ is indeed below $c_{\infty}$. As before, since $\Omega$ is bounded, then exists a ball $B_{R}(\bar{x})$ containing $\Omega$ and $\partial B_{R}(\bar{x}) \cap \bar{\Omega} \neq \emptyset$. Suppose that $x_{0} \in \partial B_{R}(\bar{x}) \cap \bar{\Omega}$, then we have $2 \alpha_{i} \geq \frac{1}{R}$ for each $1 \leq i \leq N-1$, where $2 \alpha_{i}(i=1, \cdots, N-1)$ are the principal curvatures of $\partial \Omega$ at $x_{0}$. We can suppose $x_{0}=0$ and $\Omega \subset\left\{x: x_{N}>0\right\}$ without loss of generality, then the boundary of $\Omega$ can be represented by

$$
x_{N}=h\left(x^{\prime}\right)=\Sigma_{i=1}^{N-1} \alpha_{i} x_{i}^{2}+o\left(\left|x^{\prime}\right|^{2}\right), \quad \forall x^{\prime}=\left(x_{1}, x_{2}, \cdots, x_{N-1}\right) \in D(0, \delta)
$$

for some $\delta>0$, where $D(0, \delta)=B_{\delta}(0) \cap\left\{x: x_{N}=0\right\}$.

With the above notations, we have the following estimates.

Lemma 5.2. Let $u_{\varepsilon}$ be defined by equation (5.2) and suppose $N \geq 4$, then we have

(i) $\int_{\Omega}\left|\nabla u_{\varepsilon}\right|^{2} d x=\int_{\mathbb{R}_{+}^{N}}\left|\nabla u_{\varepsilon}\right|^{2} d x-N^{\frac{N-2}{2}}(N-2)^{\frac{N+2}{2}} \varepsilon \int_{R^{N-1}} \frac{\left[\left|x^{\prime}\right|^{2}+\left(x_{N}^{0}\right)^{2}\right] g\left(x^{\prime}\right)}{\left[1+\left|x^{\prime}\right|^{2}+\left(x_{N}^{0}\right)^{2}\right]^{N}} d x^{\prime}+o(\varepsilon)$.

(ii) $\int_{\Omega} u_{\varepsilon}^{2^{*}} d x=\int_{\mathbb{R}_{+}^{N}} u_{\varepsilon}^{2^{*}} d x-N^{\frac{N}{2}}(N-2)^{\frac{N}{2}} \varepsilon \int_{R^{N-1}} \frac{g\left(x^{\prime}\right)}{\left[1+\left|x^{\prime}\right|^{2}+\left(x_{N}^{0}\right)^{2}\right]^{N}} d x^{\prime}+o(\varepsilon)$.

(iii) $\int_{\partial \Omega} u_{\varepsilon}^{2 *} d S=\int_{\mathbb{R}^{N-1}} u_{\varepsilon}^{2 *} d x^{\prime}-2(N-1) N^{\frac{N}{2}}(N-2)^{\frac{N-2}{2}} \varepsilon \int_{R^{N-1}} \frac{g\left(x^{\prime}\right)}{\left[1+\left|x^{\prime}\right|^{2}+\left(x_{N}^{0}\right)^{2}\right]^{N}} d x^{\prime}+o(\varepsilon)$.

(iv) $\int_{\Omega} u_{\varepsilon}^{2} d x= \begin{cases}O\left(\varepsilon^{2}|\ln \varepsilon|\right) & N=4, \\ O\left(\varepsilon^{2}\right) & N \geq 5\end{cases}$

Proof. The proof of this lemma is similar to the previous ones. We sketch it. For (i), a direct calculation shows that

$$
\begin{aligned}
& -\int_{\Omega}\left|\nabla u_{\varepsilon}\right|^{2} d x+\int_{\mathbb{R}_{+}^{N}}\left|\nabla u_{\varepsilon}\right|^{2} d x \\
& =N^{\frac{N-2}{2}}(N-2)^{\frac{N+2}{2}} \varepsilon^{N-2} \int_{\mathbb{R}^{N-1}} d x^{\prime} \int_{0}^{g\left(x^{\prime}\right)} \frac{\left|x^{\prime}\right|^{2}+\left(x_{N}+\varepsilon x_{N}^{0}\right)^{2}}{\left[\varepsilon^{2}+\left|x^{\prime}\right|^{2}+\left(x_{N}+\varepsilon x_{N}^{0}\right)^{2}\right]^{N}} d x_{N}+o(\varepsilon) \\
& =N^{\frac{N-2}{2}}(N-2)^{\frac{N+2}{2}} \varepsilon^{N-2} \int_{\mathbb{R}^{N-1}} \varepsilon^{N-1} d y^{\prime} \int_{0}^{\varepsilon g\left(y^{\prime}\right)} \frac{\varepsilon^{2}\left[\left|y^{\prime}\right|^{2}+\left(y_{N}+x_{N}^{0}\right)^{2}\right]}{\varepsilon^{2 N}\left[1+\left|y^{\prime}\right|^{2}+\left(y_{N}+x_{N}^{0}\right)^{2}\right]^{N}} \varepsilon d y_{N}+o(\varepsilon) \\
& =N^{\frac{N-2}{2}}(N-2)^{\frac{N+2}{2}} \varepsilon \int_{\mathbb{R}^{N-1}} \frac{\left[\left|y^{\prime}\right|^{2}+\left(x_{N}^{0}\right)^{2}\right] g\left(y^{\prime}\right)}{\left[1+\left|y^{\prime}\right|^{2}+\left(x_{N}^{0}\right)^{2}\right]^{N}} d y^{\prime}+o(\varepsilon),
\end{aligned}
$$

hence (i) follows. 
EXISTENCE RESULTS FOR SUPERLINEAR ELLIPTIC EQUATIONS WITH NONLINEAR BOUNDARY VALUE CONDITIO

Similarly, as for (ii), we have

$$
\begin{aligned}
& \int_{\mathbb{R}_{+}^{N}} u_{\varepsilon}^{2^{*}} d x-\int_{\Omega} u_{\varepsilon}^{2^{*}} d x \\
& =N^{\frac{N}{2}}(N-2)^{\frac{N}{2}} \int_{\mathbb{R}^{N-1}} d x^{\prime} \int_{0}^{g\left(x^{\prime}\right)} \frac{\varepsilon^{N}}{\left[\varepsilon^{2}+\left|x^{\prime}\right|^{2}+\left(x_{N}+\varepsilon x_{N}^{0}\right)^{2}\right]^{N}} d x_{N}+o(\varepsilon) \\
& =N^{\frac{N}{2}}(N-2)^{\frac{N}{2}} \int_{\mathbb{R}^{N-1}} \varepsilon^{N-1} d y^{\prime} \int_{0}^{\varepsilon g\left(y^{\prime}\right)} \frac{\varepsilon^{N}}{\varepsilon^{2 N}\left[1+\left|y^{\prime}\right|^{2}+\left(y_{N}+x_{N}^{0}\right)^{2}\right]^{N}} \varepsilon d y_{N}+o(\varepsilon) \\
& =N^{\frac{N}{2}}(N-2)^{\frac{N}{2}} \varepsilon \int_{\mathbb{R}^{N-1}} \frac{g\left(y^{\prime}\right)}{\left[1+\left|y^{\prime}\right|^{2}+\left(x_{N}^{0}\right)^{2}\right]^{N}} d y^{\prime}+o(\varepsilon),
\end{aligned}
$$

(ii) follows.

As for (iii), we define

$$
f(r)=u_{\varepsilon}\left(x^{\prime}, r g\left(x^{\prime}\right)\right)^{\frac{2(N-1)}{N-2}} \sqrt{1+r^{2}\left|\nabla g\left(x^{\prime}\right)\right|^{2}},
$$

then we get

$$
\begin{aligned}
& \int_{\partial \Omega} u_{\varepsilon}^{\frac{2(N-1)}{N-2}} d \sigma-\int_{\mathbb{R}^{N-1}} u_{\varepsilon}\left(x^{\prime}, 0\right)^{\frac{2(N-1)}{N-2}} d x^{\prime} \\
& =\int_{\mathbb{R}^{N-1}} u_{\varepsilon}\left(x^{\prime}, g\left(x^{\prime}\right)\right)^{\frac{2(N-1)}{N-2}} \sqrt{1+\left|\nabla g\left(x^{\prime}\right)\right|^{2}} d x^{\prime}-\int_{\mathbb{R}^{N-1}} u_{\varepsilon}\left(x^{\prime}, 0\right)^{\frac{2(N-1)}{N-2}} d x^{\prime}+o(\varepsilon) \\
& =\int_{\mathbb{R}^{N-1}} f(1)-f(0) d x^{\prime}+o(\varepsilon) \\
& =-2(N-1) N^{\frac{N-1}{2}}(N-2)^{\frac{N-1}{2}} \varepsilon^{N-1} \\
& \int_{\mathbb{R}^{N-1}} \frac{1}{\left[\varepsilon^{2}+\left|x^{\prime}\right|^{2}+\left(r_{\varepsilon} g\left(x^{\prime}\right)+\varepsilon x_{N}^{0}\right)^{2}\right]^{N}}\left[r_{\varepsilon} g\left(x^{\prime}\right)+\varepsilon x_{N}^{0}\right] g\left(x^{\prime}\right) \sqrt{1+r_{\varepsilon}^{2}\left|\nabla g\left(x^{\prime}\right)\right|^{2}} d x^{\prime}+o(\varepsilon) \\
& =-2(N-1) N^{\frac{N-1}{2}}(N-2)^{\frac{N-1}{2}} \varepsilon^{N-1} \\
& \int_{\mathbb{R}^{N-1}} \frac{\varepsilon\left[r_{\varepsilon} \varepsilon g\left(y^{\prime}\right)+x_{N}^{0}\right] \varepsilon^{2} g\left(y^{\prime}\right)}{\varepsilon^{2 N}\left[1+\left|y^{\prime}\right|^{2}+\left(r_{\varepsilon} \varepsilon g\left(y^{\prime}\right)+x_{N}^{0}\right)^{2}\right]^{N}} \sqrt{1+r_{\varepsilon}^{2} \varepsilon^{2}\left|\nabla g\left(y^{\prime}\right)\right|^{2}} \varepsilon^{N-1} d y^{\prime}+o(\varepsilon) \\
& =-2(N-1) N^{\frac{N-1}{2}}(N-2)^{\frac{N-1}{2}} \varepsilon \int_{\mathbb{R}^{N-1}} \frac{x_{N}^{0} g\left(y^{\prime}\right)}{\left[1+\left|y^{\prime}\right|^{2}+\left(x_{N}^{0}\right)^{2}\right]^{N}} d y^{\prime}+o(\varepsilon) \\
& =-2(N-1) N^{\frac{N}{2}}(N-2)^{\frac{N-2}{2}} \varepsilon \int_{\mathbb{R}^{N-1}} \frac{g\left(y^{\prime}\right)}{\left[1+\left|y^{\prime}\right|^{2}+\left(x_{N}^{0}\right)^{2}\right]^{N}} d y^{\prime}+o(\varepsilon),
\end{aligned}
$$

we get (iii).

(iv) is proved in [5]. 
Lemma 5.3. Suppose that $N \geq 4$, the the following inequality holds

$$
\sup _{t \geq 0} I\left(t u_{\varepsilon}\right)<c_{\infty}
$$

Proof. We infer from Lemma 5.2 that

$$
\begin{aligned}
I\left(t u_{\varepsilon}\right) & =\frac{t^{2}}{2} \int_{\mathbb{R}_{+}^{N}}\left|\nabla u_{\varepsilon}\right|^{2} d x-\frac{t^{2^{*}}}{2^{*}} \int_{\mathbb{R}_{+}^{N}} u_{\varepsilon}^{2^{*}} d x-\frac{t^{2 *}}{2_{*}} \int_{\mathbb{R}^{N-1}} u_{\varepsilon}\left(x^{\prime}, 0\right)^{2 *} d x^{\prime} \\
& -\frac{t^{2}}{2} N^{\frac{N-2}{2}}(N-2)^{\frac{N+2}{2}} \varepsilon \int_{\mathbb{R}^{N-1}} \frac{\left[\left|x^{\prime}\right|^{2}+\left(x_{N}^{0}\right)^{2}\right] g\left(x^{\prime}\right)}{\left[1+\left|x^{\prime}\right|^{2}+\left(x_{n}^{0}\right)^{2}\right]^{N}} d x^{\prime} \\
& +\frac{t^{2^{*}}}{2^{*}} N^{\frac{N}{2}}(N-2)^{\frac{N}{2}} \varepsilon \int_{\mathbb{R}^{N-1}} \frac{g\left(x^{\prime}\right)}{\left[1+\left|x^{\prime}\right|^{2}+\left(x_{n}^{0}\right)^{2}\right]^{N}} d x^{\prime} \\
& +\frac{t^{2 *}}{2_{*}} 2(N-1) N^{\frac{N}{2}}(N-2)^{\frac{N-2}{2}} \varepsilon \int_{\mathbb{R}^{N-1}} \frac{g\left(x^{\prime}\right)}{\left[1+\left|x^{\prime}\right|^{2}+\left(x_{n}^{0}\right)^{2}\right]^{N}} d x^{\prime}+o(\varepsilon) .
\end{aligned}
$$

We assume that $I\left(t u_{\varepsilon}\right)$ attains its maximum at $t_{\varepsilon}$, then we have $t_{\varepsilon}=1+o(1)$ as $\varepsilon \rightarrow 0$. On the other hand, we infer from the definition of $c_{\infty}$ that

$$
\begin{aligned}
& \frac{t_{\varepsilon}^{2}}{2} \int_{\mathbb{R}_{+}^{N}}\left|\nabla u_{\varepsilon}\right|^{2} d x-\frac{t_{\varepsilon}^{2^{*}}}{2^{*}} \int_{\mathbb{R}_{+}^{N}} u_{\varepsilon}^{2^{*}} d x-\frac{t_{\varepsilon}^{2 *}}{2_{*}} \int_{\mathbb{R}^{N-1}} u_{\varepsilon}\left(x^{\prime}, 0\right)^{2 *} d x^{\prime} \\
& \leq \frac{1}{2} \int_{\mathbb{R}_{+}^{N}}\left|\nabla u_{\varepsilon}\right|^{2} d x-\frac{1}{2^{*}} \int_{\mathbb{R}_{+}^{N}} u_{\varepsilon}^{2^{*}} d x-\frac{1}{2_{*}} \int_{\mathbb{R}^{N-1}} u_{\varepsilon}\left(x^{\prime}, 0\right)^{2 *} d x^{\prime} \\
& =c_{\infty} .
\end{aligned}
$$

Hence, to show $I\left(t_{\varepsilon} u_{\varepsilon}\right)<c_{\infty}$, it is sufficient to show that

$$
\begin{aligned}
& -\frac{1}{2} N^{\frac{N-2}{2}}(N-2)^{\frac{N+2}{2}} \int_{\mathbb{R}^{N-1}} \frac{\left[\left|x^{\prime}\right|^{2}+\left(x_{N}^{0}\right)^{2}\right] g\left(x^{\prime}\right)}{\left[1+\left|x^{\prime}\right|^{2}+\left(x_{N}^{0}\right)^{2}\right]^{N}} d x^{\prime} \\
& +\frac{1}{2} N^{\frac{N-2}{2}}(N-2)^{\frac{N+2}{2}} \int_{\mathbb{R}^{N-1}} \frac{g\left(x^{\prime}\right)}{\left[1+\left|x^{\prime}\right|^{2}+\left(x_{n}^{0}\right)^{2}\right]^{N}} d x^{\prime} \\
& +N^{\frac{N}{2}}(N-2)^{\frac{N}{2}} \int_{\mathbb{R}^{N-1}} \frac{g\left(x^{\prime}\right)}{\left[1+\left|x^{\prime}\right|^{2}+\left(x_{n}^{0}\right)^{2}\right]^{N}} d x^{\prime}<0 .
\end{aligned}
$$

We need to compare the value

$$
\int_{\mathbb{R}^{N-1}} \frac{\left|x^{\prime}\right|^{2} g\left(x^{\prime}\right)}{\left[1+\left|x^{\prime}\right|^{2}+\left(x_{n}^{0}\right)^{2}\right]^{N}} d x^{\prime}
$$

with

$$
\int_{\mathbb{R}^{N-1}} \frac{g\left(x^{\prime}\right)}{\left[1+\left|x^{\prime}\right|^{2}+\left(x_{n}^{0}\right)^{2}\right]^{N}} d x^{\prime}
$$


EXISTENCE RESULTS FOR SUPERLINEAR ELLIPTIC EQUATIONS WITH NONLINEAR BOUNDARY VALUE CONDITIO If we denote $c=1+\left(x_{N}^{0}\right)^{2}=\frac{2(N-1)}{N-2}$, then a direct calculation shows that

$$
\begin{aligned}
& \int_{\mathbb{R}^{N-1}} \frac{\left|x^{\prime}\right|^{2} g\left(x^{\prime}\right)}{\left[1+\left|x^{\prime}\right|^{2}+\left(x_{n}^{0}\right)^{2}\right]^{N}} d x^{\prime} \\
& =\int_{\mathbb{R}^{N-1}} \frac{\left|x^{\prime}\right|^{2}\left(\sum_{i=1}^{N-1} \alpha_{i} x_{i}^{2}\right)}{\left[c+\left|x^{\prime}\right|^{2}\right]^{N}} d x^{\prime} \\
& =\left(\sum_{i=1}^{N-1} \alpha_{i}\right) \int_{\mathbb{R}^{N-1}} \frac{\left|x^{\prime}\right|^{2} x_{i}^{2}}{\left[c+\left|x^{\prime}\right|^{2}\right]^{N}} d x^{\prime} \\
& =\frac{1}{2} H(0) \int_{\mathbb{R}^{N-1}} \frac{\left|x^{\prime}\right|^{4}}{\left[c+\left|x^{\prime}\right|^{2}\right]^{N}} d x^{\prime} \\
& =\frac{1}{2} H(0) \omega_{N-2} \int_{0}^{\infty} \frac{r^{N+2}}{\left[c+r^{2}\right]^{N}} d r .
\end{aligned}
$$

Similarly, we have

$$
\begin{aligned}
& \int_{\mathbb{R}^{N-1}} \frac{g\left(x^{\prime}\right)}{\left[1+\left|x^{\prime}\right|^{2}+\left(x_{n}^{0}\right)^{2}\right]^{N}} d x^{\prime} \\
& =\int_{\mathbb{R}^{N-1}} \frac{\left(\sum_{i=1}^{N-1} \alpha_{i} x_{i}^{2}\right)}{\left[c+\left|x^{\prime}\right|^{2}\right]^{N}} d x^{\prime} \\
& =\left(\sum_{i=1}^{N-1} \alpha_{i}\right) \int_{\mathbb{R}^{N-1}} \frac{x_{i}^{2}}{\left[c+\left|x^{\prime}\right|^{2}\right]^{N}} d x^{\prime} \\
& =\frac{1}{2} H(0) \int_{\mathbb{R}^{N-1}} \frac{\left|x^{\prime}\right|^{2}}{\left[c+\left|x^{\prime}\right|^{2}\right]^{N}} d x^{\prime} \\
& =\frac{1}{2} H(0) \omega_{N-2} \int_{0}^{\infty} \frac{r^{N}}{\left[c+r^{2}\right]^{N}} d r .
\end{aligned}
$$

On the other hand, we have

$$
\begin{aligned}
\int_{0}^{\infty} \frac{r^{N+2}}{\left[c+r^{2}\right]^{N}} d r & =\int_{0}^{\infty} \frac{\left(c+r^{2}\right) r^{N}}{\left[c+r^{2}\right]^{N}} d r-c \int_{0}^{\infty} \frac{r^{N}}{\left[c+r^{2}\right]^{N}} d r \\
& =\frac{2(N-1)}{N+1} \int_{0}^{\infty} \frac{r^{N+2}}{\left[c+r^{2}\right]^{N}} d r-\frac{2(N-1)}{N-2} \int_{0}^{\infty} \frac{r^{N}}{\left[c+r^{2}\right]^{N}} d r
\end{aligned}
$$

which implies

$$
\int_{0}^{\infty} \frac{r^{N+2}}{\left[c+r^{2}\right]^{N}} d r=\frac{2(N-1)(N+1)}{(N-2)(N-3)} \int_{0}^{\infty} \frac{r^{N}}{\left[c+r^{2}\right]^{N}} d r
$$


Inserting this equation into the left hand side of equation (5.11), then we get

$$
\begin{aligned}
& -\frac{1}{2} N^{\frac{N-2}{2}}(N-2)^{\frac{N+2}{2}} \int_{\mathbb{R}^{N-1}} \frac{\left[\left|x^{\prime}\right|^{2}+\left(x_{N}^{0}\right)^{2}\right] g\left(x^{\prime}\right)}{\left[1+\left|x^{\prime}\right|^{2}+\left(x_{N}^{0}\right)^{2}\right]^{N}} d x^{\prime} \\
& +\frac{1}{2} N^{\frac{N-2}{2}}(N-2)^{\frac{N+2}{2}} \int_{\mathbb{R}^{N-1}} \frac{g\left(x^{\prime}\right)}{\left[1+\left|x^{\prime}\right|^{2}+\left(x_{n}^{0}\right)^{2}\right]^{N}} d x^{\prime} \\
& +N^{\frac{N}{2}}(N-2)^{\frac{N}{2}} \int_{\mathbb{R}^{N-1}} \frac{g\left(x^{\prime}\right)}{\left[1+\left|x^{\prime}\right|^{2}+\left(x_{n}^{0}\right)^{2}\right]^{N}} d x^{\prime} \\
& =\frac{\omega_{N-2}}{2} N^{\frac{N-2}{2}}(N-2)^{\frac{N}{2}} H(0)\left[-\frac{(N-1)(N+1)}{N-3}-\frac{N}{2}+\frac{N-2}{2}+N\right] \int_{0}^{\infty} \frac{r^{N}}{\left(c+r^{2}\right)^{N}} d r \\
& =-2 N^{\frac{N-2}{2}}(N-2)^{\frac{N}{2}} \frac{N-1}{N-3} H(0) \omega_{N-2} \int_{0}^{\infty} \frac{r^{N}}{\left(c+r^{2}\right)^{N}} d r \\
& <0
\end{aligned}
$$

since $H(0)>0$. This proves equation (5.11). Hence we deduce that

$$
I\left(t_{\varepsilon} u_{\varepsilon}\right)<0
$$

for $\varepsilon$ small enough.

Finally, we study the case $N=3$. In this case, we suppose the principal curvatures of $\partial \Omega$ at $x_{0} \in \partial \Omega$ belong to interval $(2 a, 2 A)$ for some $0<a \leq A<\infty$. Then we have $a\left|x^{\prime}\right|^{2} \leq h\left(x^{\prime}\right) \leq A\left|x^{\prime}\right|^{2}$ for $x^{\prime} \in D(0, \delta)$. With these notations, we have the following estimates.

Lemma 5.4. Let $N=3, \Omega$ as above and $u_{\varepsilon}$ be defined by equation (5.2), then we have

(i) $\int_{\Omega}\left|\nabla u_{\varepsilon}\right|^{2} d x \leq \int_{\mathbb{R}_{+}^{N}}\left|\nabla u_{\varepsilon}\right|^{2} d x-C \varepsilon|\ln \varepsilon|+O(\varepsilon)$.

(ii) $\int_{\Omega} u_{\varepsilon}^{6} d x \geq \int_{\mathbb{R}_{+}^{N}} u_{\varepsilon}^{6} d x-C \varepsilon$.

(iii) $\int_{\partial \Omega} u_{\varepsilon}^{4} d \sigma=\int_{\mathbb{R}^{N-1}} u_{\varepsilon}^{4} d x^{\prime}-C \varepsilon$.

(iv) $\int_{\Omega} u_{\varepsilon}^{2}=O(\varepsilon)$.

Proof. As for (i), we have

$$
\begin{aligned}
\int_{\Omega}\left|\nabla u_{\varepsilon}\right|^{2} d x & =\int_{\mathbb{R}_{+}^{N}}\left|\nabla u_{\varepsilon}\right|^{2} d x-\int_{D(0, \delta)} d x^{\prime} \int_{0}^{h\left(x^{\prime}\right)}\left|\nabla u_{\varepsilon}\right|^{2} d x_{N}+O\left(\varepsilon^{N-2}\right) \\
& \leq \int_{\mathbb{R}_{+}^{N}}\left|\nabla u_{\varepsilon}\right|^{2} d x-\int_{D(0, \delta)} d x^{\prime} \int_{0}^{a\left|x^{\prime}\right|^{2}}\left|\nabla u_{\varepsilon}\right|^{2} d x_{N}+O(\varepsilon) .
\end{aligned}
$$


EXISTENCE RESULTS FOR SUPERLINEAR ELLIPTIC EQUATIONS WITH NONLINEAR BOUNDARY VALUE CONDITIO

For the second term on the right hand of the above equation, we have

$$
\begin{aligned}
& \int_{D(0, \delta)} d x^{\prime} \int_{0}^{a\left|x^{\prime}\right|^{2}}\left|\nabla u_{\varepsilon}\right|^{2} d x_{N} \\
& \geq N^{\frac{1}{2}}(N-2)^{\frac{5}{2}} \varepsilon \int_{D(0, \delta)} d x^{\prime} \int_{0}^{a\left|x^{\prime}\right|^{2}} \frac{\left|x^{\prime}\right|^{2}+\left(x_{N}+\varepsilon x_{N}^{0}\right)^{2}}{\left[\varepsilon^{2}+\left|x^{\prime}\right|^{2}+\left(x_{N}+\varepsilon x_{N}^{0}\right)^{2}\right]^{3}} d x_{N} \\
& =C \int_{D\left(0, \varepsilon^{-1} \delta\right)} d x^{\prime} \int_{0}^{a \varepsilon\left|x^{\prime}\right|^{2}} \frac{\left|x^{\prime}\right|^{2}+\left(x_{N}+x_{N}^{0}\right)^{2}}{\left[1+\left|x^{\prime}\right|^{2}+\left(x_{N}+x_{N}^{0}\right)^{2}\right]^{3}} d x_{N} \\
& \geq C \int_{D\left(0, \varepsilon^{-1} \delta\right)} \frac{\left|x^{\prime}\right|^{2}+\left(x_{N}^{0}\right)^{2}}{\left[1+\left|x^{\prime}\right|^{2}+\left(x_{N}+x_{N}^{0}\right)^{2}\right]^{3}} \varepsilon\left|x^{\prime}\right|^{2} d x^{\prime} \\
& =C \varepsilon \int_{0}^{\varepsilon^{-1} \delta} \frac{r^{2}+\left(x_{N}^{0}\right)^{2}}{\left[1+r^{2}+\left(x_{N}^{0}\right)^{2}\right]^{3}} r^{3} d r \\
& =C \varepsilon\left[\int_{0}^{\sqrt{1+\left(x_{N}^{0}\right)^{2}}} \frac{r^{5}}{\left[1+r^{2}+\left(x_{N}^{0}\right)^{2}\right]^{3}} d r+\int_{\sqrt{1+\left(x_{N}^{0}\right)^{2}}}^{\varepsilon^{-1} \delta} \frac{r^{5}}{\left[1+r^{2}+\left(x_{N}^{0}\right)^{2}\right]^{3}} d r\right. \\
& \left.+\int_{0}^{\varepsilon^{-1} \delta} \frac{\left(x_{N}^{0}\right)^{2} r^{3}}{\left[1+r^{2}+\left(x_{N}^{0}\right)^{2}\right]^{3}} d r\right] \\
& \geq C \varepsilon[|\ln \varepsilon|+C] .
\end{aligned}
$$

Hence, (i) follows.

For (ii), we first note that

$$
\int_{\Omega} u_{\varepsilon}^{6}=\int_{\mathbb{R}^{N-1}} u_{\varepsilon}^{6} d x-\int_{D(0, \delta)} d x^{\prime} \int_{0}^{h\left(x^{\prime}\right)} u_{\varepsilon}^{6} d x_{N}+O\left(\varepsilon^{3}\right) .
$$

While a direct calculation shows that

$$
\begin{aligned}
& \int_{D(0, \delta)} d x^{\prime} \int_{0}^{h\left(x^{\prime}\right)} h_{\varepsilon}^{6} d x_{N} \\
& \leq C \int_{D(0, \delta)} \int_{0}^{A\left|x^{\prime}\right|^{2}} \frac{\varepsilon^{3}}{\left[\varepsilon^{2}+\left|x^{\prime}\right|^{2}+\left(x_{N}+\varepsilon x_{N}^{0}\right)^{2}\right]^{3}} d x_{N} \\
& =C \int_{D\left(0, \varepsilon^{-1} \delta\right)} d x^{\prime} \int_{0}^{\varepsilon A\left|x^{\prime}\right|^{2}} \frac{1}{\left[1+\left|x^{\prime}\right|^{2}+\left(x_{N}+x_{N}^{0}\right)^{2}\right]^{3}} d x_{N} \\
& \leq C \int_{D\left(0, \varepsilon^{-1} \delta\right)} \frac{A \varepsilon\left|x^{\prime}\right|^{2}}{\left[c+\left|x^{\prime}\right|^{2}\right]^{3}} d x^{\prime} \\
& =C \varepsilon \int_{0}^{\varepsilon^{-1} \delta} \frac{r^{3}}{\left[c+r^{2}\right]^{3}} d r \\
& =C \varepsilon
\end{aligned}
$$

hence (ii) follows. 
For (iii), if we define

$$
f(r)=u_{\varepsilon}\left(x^{\prime}, r g\left(x^{\prime}\right)\right)^{4} \sqrt{1+r^{2}\left|\nabla g\left(x^{\prime}\right)\right|^{2}},
$$

then we have

$$
\begin{aligned}
& \int_{\partial \Omega} u_{\varepsilon}^{4}-\int_{\mathbb{R}^{N-1}} u_{\varepsilon}\left(x^{\prime}, 0\right)^{4} d x^{\prime} \\
& =\int_{\mathbb{R}^{N-1}} f(1)-f(0) d x^{\prime}+O\left(\varepsilon^{2}\right) \\
& =C \int_{\mathbb{R}^{N-1}} u_{\varepsilon}\left(x^{\prime}, r_{\varepsilon} g\left(x^{\prime}\right)\right)^{3} \frac{\partial u_{\varepsilon}}{\partial x_{N}}\left(x^{\prime}, r_{\varepsilon} g\left(x^{\prime}\right)\right) g\left(x^{\prime}\right) \sqrt{1+r_{\varepsilon}^{2}\left|\nabla g\left(x^{\prime}\right)\right|^{2}} d x^{\prime}+o(\varepsilon) \\
& =-C \int_{\mathbb{R}^{N-1}} \frac{\varepsilon^{2}}{\left[\varepsilon^{2}+\left|x^{\prime}\right|^{2}+\left(r_{\varepsilon} g\left(x^{\prime}\right)+\varepsilon x_{N}^{0}\right)^{2}\right]^{3}}\left(r_{\varepsilon} g\left(x^{\prime}\right)+\varepsilon x_{N}^{0}\right) g\left(x^{\prime}\right) \sqrt{1+r_{\varepsilon}^{2}\left|\nabla g\left(x^{\prime}\right)\right|^{2}} d x^{\prime}+o(\varepsilon) \\
& =-C \varepsilon \int_{\mathbb{R}^{N-1}} \frac{g\left(x^{\prime}\right)}{\left[1+\left|x^{\prime}\right|^{2}+\left(x_{N}^{0}\right)^{2}\right]^{3}} d x^{\prime}+o(\varepsilon) \\
& =C \varepsilon+o(\varepsilon),
\end{aligned}
$$

hence (iii) follows.

(iv) is proved in [5].

With the above preparations, we can estimate the Mountain Pass level of $I$ for $N=3$ now. More precisely, we have the following result.

Lemma 5.5. Suppose that $N=3$, then we have

$$
\sup _{t \geq 0} I\left(t u_{\varepsilon}\right)<c_{\infty}
$$

for $\varepsilon$ small enough.

Proof. We infer from Lemma 5.4 that

$$
\begin{aligned}
I\left(t u_{\varepsilon}\right) & =\frac{t^{2}}{2} \int_{\mathbb{R}_{+}^{N}}\left|\nabla u_{\varepsilon}\right|^{2} d x-\frac{t^{2^{*}}}{2^{*}} \int_{\mathbb{R}_{+}^{N}} u_{\varepsilon}^{2^{*}} d x-\frac{t^{2 *}}{2_{*}} \int_{\mathbb{R}^{N-1}} u_{\varepsilon}^{2_{*}} d x^{\prime} \\
& -\frac{t^{2}}{2} C \varepsilon|\ln \varepsilon|+\frac{t^{2^{*}}}{2^{*}} C \varepsilon+\frac{t^{2 *}}{2_{*}} C \varepsilon+O(\varepsilon) .
\end{aligned}
$$

Suppose that $I\left(t u_{\varepsilon}\right)$ attains its maximum at $t_{\varepsilon}$, then it is easy to see that $t_{\varepsilon}=1+o(1)$ as $\varepsilon \rightarrow 0$. Moreover, we have

$$
\begin{aligned}
& \frac{t_{\varepsilon}^{2}}{2} \int_{\mathbb{R}_{+}^{N}}\left|\nabla u_{\varepsilon}\right|^{2} d x-\frac{t_{\varepsilon}^{2^{*}}}{2^{*}} \int_{\mathbb{R}_{+}^{N}} u_{\varepsilon}^{2^{*}} d x-\frac{t_{\varepsilon}^{2_{*}}}{2_{*}} \int_{\mathbb{R}^{N-1}} u_{\varepsilon}\left(x^{\prime}, 0\right)^{2_{*}} d x^{\prime} \\
& \leq \frac{1}{2} \int_{\mathbb{R}_{+}^{N}}\left|\nabla u_{\varepsilon}\right|^{2} d x-\frac{1}{2^{*}} \int_{\mathbb{R}_{+}^{N}} u_{\varepsilon}^{2^{*}} d x-\frac{1}{2_{*}} \int_{\mathbb{R}^{N-1}} u_{\varepsilon}\left(x^{\prime}, 0\right)^{2_{*}} d x^{\prime} \\
& =c_{\infty} .
\end{aligned}
$$


Insert this equation into equation (5.20), then we get

$$
I\left(t_{\varepsilon} u_{\varepsilon}\right)<c_{\infty}
$$

for $\varepsilon$ small enough. So the conclusion of this lemma follows.

Proof of Theorem 1.4. The proof of Theorem 1.4 is a direct consequence of the Mountain Pass theorem. In fact, it is easy to see that the functional $I$ possesses the Mountain Pass structure, so the Mountain Pass value $c$ is well-defined. Moreover, it follows from Lemma 5.1 that $I$ satisfies the $(P S)_{c}$ condition for $c<c_{\infty}$. Finally, we infer from Lemma 5.3 and Lemma 5.5 that $c<c_{\infty}$. So $c$ is a nontrivial critical value for functional $I$, that is, problem (1.1) possesses a nontrivial solution under the assumptions of Theorem 1.4.

Acknowledgement: This work is supported by NSFC, No.11101291.

\section{REFERENCES}

[1] B.Barrios, E.Colorado, A.de Pablo, U.Sánchez, On some critical problems for the fractional Laplacian oprator, J. Differential Equations, 252(2012), 6133-6162.

[2] J.Bonder, J.Rossi, Existence results for the p-Laplacian with nonlinear boundary conditions, J. Math. Anal. Appl., 263(2001), 195-223.

[3] C.Brãndle, E.Colorado, A.de Pablo, U. Sánchez, A concave-convex elliptic problem involving the fractional Laplacian, Proc. Roy. Soc. Edinburgh Sect. A., 143(2013), 39-71.

[4] H.Brezis, E.Lieb, A relation between pointwise convergence of functions and convergence of functionals, Proc. Amer. Math. Soc., 88(1983), 486-490.

[5] H.Brezis, L.Nirenberg, Positive solutions of nonlinear elliptic equations involving critical Sobolev exponents, Comm. Pure. Appl. Math., 3(1989), 437-477.

[6] X.Cabre and J.Tan, Positive solutions of nonlinear problems involving the square root of the Laplacian, Advances in Mathematics, 224(2010), 2052-2093.

[7] L.Caffarelli, L. Silvestre, An extension problem related to the fractional Laplacian, Comm. Partial Diff. Euqas., 32 (2007), 1245-1260.

[8] A.Capozzi, D.Fortunato and G. Palmieri, An existence result for nonlinear elliptic problems involving critical Sobolev exponent, Ann. I. H. Poincare,6(1985), 463-470.

[9] M.Chipot, M.Chlebik, M.Fila, I.Shafrir, Existence of positive solutions of a semilinear elliptic equation in $R_{+}^{n}$ with a nonlinear boundary condition, J. Math. Anal. Appl., 223 (1998), 429-471.

[10] M.Chipot, I.Shafrir, M.Fila, On the solutions to some elliptic equations with nonlinear Neumann boundary conditions, Adv. Differential Equations, 1(1996), 91-110.

[11] Y.Ge, J.Wei, F.Zhou, A critical elliptic problem for polyharmonic operators, J. Funct. Anal., 260(2011),

[12] B.Hu, Nonexistence of a positive solution of the Laplace equation with a nonlinear boundary condition, Differential Integral Equations, 7(1994), 301-313.

[13] B.Hu, H.Yin, On critical exponents for the heat equation with a mixed nonlinear Dirichlet-Neumann boundary condition, J. Math. Anal. Appl., 209(1997), 683-711.

[14] Y.Hua, X.Yu, On the ground state solution for a critical fractional Laplacian equation, Nonlinear Anal., 87(2013), 116-125.

[15] B.Hu, H.Yin, On critical exponents for the heat equation with a nonlinear boundary condition, Ann. Inst. H. Poincare Anal. Non Lineaire, 13(1996), 707-732. 
[16] B.Hu, H.Yin, The profile near blowup time for solution of the heat equation with a nonlinear boundary condition, Trans. Amer. Math. Soc., 346(1994), 117-135.

[17] Y.Hua, X.Yu, On the ground state solution for a critical fractional Laplacian equation, Nonlinear Anal., 87(2013), 116-125.

[18] Y.Li, L.Zhang, Liouville-type theorems and harnack-type inequalities for semilinear elliptic equations,Journal d'Analyse Mathmatique, 90(2003), 27-87.

[19] Y.Li, M.Zhu, Uniqueness theorems through the method of moving spheres, Duke Math. J., 80(1995), 383-417.

[20] P.H.Rabinowitz, Minimax Methods in Critical Point Theory with Applications to Differential Equations, CBMS Regional Conference, 65, AMS, Providence, R.I., 1986.

[21] J.Tan, The Brezis-Nirenberg type problem involving the square root of the Laplacian, Calc. Var. Partial Differential Equations, 42(2011), 21-41.

[22] X.Wang, Neumann problems of semilinear elliptic equations involving critical Sobolev exponents, J. Differential Equations, 93(1991), 283-310.

[23] Y.Ye, X.Yu, A global compactness result for a critical semilinear elliptic equation in $R^{N}$, Nonlinear Anal., 71 (2009), 1844-1849.

[24] X.Yu, Liouville Type Theorem for Nonlinear Elliptic Equation with General Nonlinearity, Discrete and Continuous Dynamical Systems-Series A, 34(2014), 4947C4966. 